Research Paper

\title{
OTUDI stabilizes PTEN to inhibit the PI3K/AKT and TNF-alpha/NF-kappaB signaling pathways and sensitize ccRCC to TKIs
}

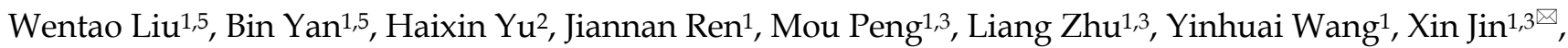

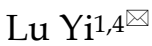 \\ 1. Department of Urology, The Second Xiangya Hospital, Central South University, Changsha, Hunan, 410011, China \\ 2. 2Cancer center, Union Hospital, Tongji Medical College, Huazhong University of Science and Technology, Wuhan, 430022, China \\ 3. 3Uro-Oncology Institute of Central South University, Changsha, Hunan, 410011, China \\ 4. 4Hunan Engineering Research Center of Smart and Precise Medicine, Changsha, Hunan, 410011, China \\ 5. 5These authors contributed equally. \\ $\square$ Corresponding author: Xin Jin (jinxinxy2@csu.edu.cn), Lu Yi (yilu9999@csu.edu.cn) \\ (c) The author(s). This is an open access article distributed under the terms of the Creative Commons Attribution License (https://creativecommons.org/licenses/by/4.0/). \\ See http://ivyspring.com/terms for full terms and conditions.
}

Received: 2021.11.21; Accepted: 2022.01.16; Published: 2022.01.24

\begin{abstract}
Clear cell renal cell carcinoma (ccRCC) is the most common subtype of renal cell carcinoma and has the highest mortality rate. For metastatic RCC, systemic drug therapy is the most important method in addition to surgical tumor reduction. In recent years, tyrosine kinase inhibitors (TKIs) targeting the angiogenesis have been applied to treat $\mathrm{ccRCC}$ and achieved profound therapeutic effects. It has been reported that most patients receiving antiangiogenic therapy will develop resistance within 15 months. The mechanism of resistance to targeted therapy is extremely complex and has not been clarified. Ovarian tumor-associated protease domain-containing proteins (OTUDs) belonging to DUBs play a critical role in the tumorigenesis of solid tumors. However, the specific role of OTUDs in ccRCC is still elusive. Here, we investigated the clinicopathological role of OTUD family members in ccRCC. We demonstrated that OTUDI was downregulated in renal cancer and involved in the poor prognosis of renal cancer. Then, we showed that OTUDI inhibits cancer cell growth. Moreover, analysis of OTUD1 RNA-seq data indicated that OTUDI inhibition triggers the AKT and NF-kappa B pathways in renal cancer cells. Furthermore, OTUDI interacts with PTEN and regulates its stability. Subsequently, we revealed that downregulation of OTUDI contributes to the sensitivity of renal cancer cells to TKIs, and this effect was blocked by TNF/NF-kappa B inhibitors and AKT inhibitors. Thus, we identified that the OTUDI-PTEN axis suppresses tumor growth and regulates the resistance of renal cancer to TKIs.
\end{abstract}

Key words: ccRCC, OTUD1, PTEN, TKIs

\section{Introduction}

Renal cell carcinoma (RCC) is a common urologic malignancy [1]. Its incidence and mortality rates rank fifteenth among those of all malignant tumors [2]. Clear cell renal cell carcinoma (ccRCC) is the most common subtype of RCC and has the highest mortality rate [3]. According to clinical stage, RCC is divided into localized RCC (T1-T2), locally advanced RCC (T3-T4) and metastatic RCC (M1); approximately $16 \%$ of RCCs are metastatic [2]. The 5-year survival rate of localized RCC is approximately $93 \%$, while that of metastatic RCC is only $12 \%$ [2]. Surgical resection is the main treatment for RCC [4]. For metastatic RCC, systemic drug therapy is the most important method in addition to surgical tumor reduction [5]. In recent years, targeted drugs and immune checkpoint inhibitors have been applied to treat ccRCC and have achieved profound therapeutic effects [6]. Exploration of the mechanism by which ccRCC develops is crucial for identifying new targets to prolong the survival time of patients with ccRCC.

The von Hippel-Lindau (VHL) gene mutation (present in approximately $90 \%$ of renal cancer 
patients) results in the excessive vascularization of tumor tissues [7]. Antiangiogenesis therapy, including VEGF (bevacizumab), tyrosine kinase inhibitors (TKIs), and mTOR inhibitors (everolimus), has become the standard therapy for patients with renal cancer [8]. It has been reported that most patients receiving antiangiogenic therapy will develop resistance within 15 months [9]. The mechanism of resistance to targeted therapy is extremely complex and has not been clarified.

Ubiquitination is a reversible process regulated by the ubiquitin-proteasome system, which is important for the homeostasis of cells [10]. Deubiquitinating enzymes (DUBs) remove the ubiquitination modification from the target protein and play an important role in the ubiquitinproteasome system. DUBs are closely correlated with the sensitivity of antitumor drugs [11]. Ovarian tumor-associated protease domain-containing proteins (OTUDs) belonging to DUBs play a critical role in the tumorigenesis of solid tumors [12]. However, the specific role of OTUDs in ccRCC is still elusive. In the current study, we investigated the clinicopathological role of OTUD family members in ccRCC. We showed that OTUD1 was closely associated with an unfavorable prognosis in ccRCC. Then, we found that OTUD1 participates in inhibiting cell proliferation and inactivating the PI3K/AKT and TNF-alpha/NF-kappa B signaling pathways in ccRCC. Moreover, we revealed that OTUD1 stabilizes PTEN, which is a negative regulator of both the PI3K/AKT and TNF-alpha/NF-kappa B signaling pathways [13]. We proved that OTUD1 regulates the resistance of TKIs in ccRCC via the NF-kappa B pathway. Therefore, we showed that OTUD1 loss contributes to the resistance of TKIs through PTEN in ccRCC.

\section{Methods and material}

\section{Cell lines and cell culture}

The two ccRCC cell lines that we cultured, 786-O and $\mathrm{ACHN}$, were obtained from Yuchi Biology (Shanghai, China), and both cell lines were identified by short tandem repeat (STR) profiling. Cells were cultured in RPMI-1640 medium (Gibco, USA) or minimum essential medium (MEM, Gibco, USA) supplemented with $10 \%$ fetal bovine serum (FBS; AC03L055, Shanghai Life-iLab Biotech, China) and 1\% penicillin, placed in an incubator at $37^{\circ} \mathrm{C}$ in $5 \% \mathrm{CO}$.

\section{Antibodies, chemical agents, siRNAs and plasmids}

The antibodies used for western blot analysis are as follows: GAPDH (\#60004-1-Ig, Proteintech, 1:5000 dilution), OTUD1 (\#bs-17563R, Bioss antibodies, 1:2000 dilution), PTEN (\#22034-1-AP, Proteintech, 1:1000 dilution), AKT (\#bsm-33278M, Bioss antibodies, 1:2000 dilution), pAKT-S473 (\#4060S, Cell signaling technology,1:1000 dilution), cleaved caspase 3 (\#9661, Cell signaling technology,1:2000 dilution), Caspase 3 (\#19677-1-AP, Proteintech, 1:1000 dilution). MG132 (\#S2619), Sunitinib (\#S7781), Pazopanib (\#S3021), JSH-23 (\#S7351), MK2206 (\#S1078), GSK1120212 (\#S2673), and LY294002 (\#S1105) were purchased from Selleckchem (Shanghai, China). HA-OTUD1 was constructed by cloning the cDNA of OTUD1 into the OmicLinkTM Expression Clone (CMV Promoter) (GeneCopoeia, EX-V0006-M14, USA). The siRNAs were purchased from RiboBio. The sequences of the siRNAs were provided in Table S1. Cells were transfected with the indicated plasmids or siRNAs using Lipofectamine 2000 (Thermo Fisher Scientific, China) according to the manufacturer's instructions. Caspase 3 activity assay kit (\#ab39401) was purchases from Abcam.

\section{Coimmunoprecipitation and Western blot analysis}

The detail of coimmunoprecipitation (IP) was reported previously [14]. In brief, cell proteins were lysed in the RIPA buffer (\#P0013, Beyotime, China) and added with protein A+G beads (\#P2029, Beyotime, China) and IgG (\#A7007, Beyotime, China) or a primary antibody. The next day, the beads were washed 6 times with RIPA buffer, and $60 \mu \mathrm{l}$ of 1X loading buffer was added. Finally, the beads were boiled and subjected to Western blotting. For Western blotting analysis, the boiled protein lysates were electrophoresed on SDS-PAGE gels. Protein expression levels were measured by using ImageJ software (National Institutes of Health, USA).

\section{Real time RT-PCR analysis}

Total RNA from cells was extracted using TRIzol reagent (\#AG21102, Accurate Biotechnology, Hunan, China). The reverse transcription kits (\#AG11728, Accurate Biotechnology, Hunan, China) and PCR kits (\#AG11701, Accurate Biotechnology, Hunan, China) was used to perform RT-qPCR according to the manufacturer's instructions. All values were normalized to the corresponding GAPDH values, and the $2-\Delta \Delta \mathrm{Ct}$ method was used to quantify the fold change. The sequences of primers were provided in Table S2.

\section{Cell proliferation assay}

For Cell Counting Kit-8 (CCK-8) assay, CCK-8 reagent (\#C0037, Beyotime) was added to each cell well and an absorbance of $450 \mathrm{~nm}$ was measured with a microplate reader. 
All animal experiments were approved by the ethics committee of the Second Xiangya Hospital, Central South University (Animal license number: 2021897). BALB/c nude mice (6 weeks old) were purchased from Vital River (Beijing, China). Cells were subcutaneously injected into the left side of the backs of the mice $\left(1 \times 10^{7}\right.$ cells per mouse). Tumor volume was calculated using the formula $\left(\mathrm{L} \times \mathrm{W}^{2}\right) / 2$. Once the mice were euthanized, the tumors were excised and weighed.

\section{Tissue microarray and immunohistochemistry (IHC)}

The tissue microarray slides (\# U081ki01) were purchased from bioaitech, China. The tissue microarray specimens were immunostained with OTUD1 and PTEN. The method of scoring of staining intensity was mentioned previously $[14,15]$.

\section{Cell cycle and Annexin v-FITC/PI assay}

Cells were washed three times with PBS and resuspended in Binding Buffer. For cell cycle analysis, cells were stained with Propidium iodide (PI). For Annexin v-FITC/PI assay, cells were stained with Annexin v-FITC and PI following the manufacturer's instruction of Annexin V-FITC Apoptosis Detection Kit (Solarbio life science, Beijing, China). Cells were incubated for $15 \mathrm{~min}$ at room temperature and analyzed on a flow cytometer (FACSCalibur, Becton, Dickinson and Company, USA). Data was analyzed with FlowJo analysis software.

\section{RNA sequencing analysis}

The 786-O cells were transfected with siNC or siOTUD1 for $48 \mathrm{~h}$. These cells were subjected to RNA sequencing analysis as previously reported [16]. The RNA sequencing analysis was performed by Novogene (Beijing, China). Briefly, a total of $1 \mu \mathrm{g}$ of RNA per sample was used as the starting material for RNA. sequencing (RNA-seq). Sequencing libraries were generated using the NEBNext Ultra RNA Library Prep Kit for Illumina (NEB, USA) following the manufacturer's instructions, and index codes were added to attribute sequences to each sample. Clustering of the samples was performed on the cBot Cluster Generation System using the TruSeq PE Cluster Kit v3-cBot-HS (Illumina) according to the manufacturer's instructions. After cluster generation, libraries were sequenced on an Illumina Novaseq platform, and $150 \mathrm{bp}$ paired-end reads were generated. FeatureCounts v1.5.0-p3 was used to count the read numbers mapped to each gene. Differential expression analysis was performed using the DESeq2
$\mathrm{R}$ package (1.16.1), and the clusterProfiler $\mathrm{R}$ package was used to test the statistical enrichment of differentially expressed genes (DEGs) in KEGG (Kyoto Encyclopedia of Genes and Genomes) pathways.

\section{Statistical analysis}

Data were expressed as means \pm SD. Statistical significance was determined by one-way or two-way ANOVA using GRAPHPAD PRISM 5 software, San Diego, CA, USA. A statistical significance threshold of P-values $<0.05$ was used.

Other methods were provided in the supplementary information.

\section{Results}

OTUD 1 is downregulated and correlated with an unfavorable prognosis in CCRCC patients

First, we studied the clinicopathological role of OTUD family members in ccRCC by analyzing The Cancer Genome Atlas (TCGA) kidney renal clear cell carcinoma (KIRC) dataset. We found that OTUD3, OTUD6A and OTUD7C were upregulated, but OTUD1, OTUD2, OTUD6B and OTUD7B were downregulated in KIRC tissues compared to normal renal tissues (Fig. 1A). Among the upregulated OTUD family members, there were no risk-related genes with hazard ratios (HRs) greater than 1 and $P$ values less than 0.05 (Fig. 1A). Notably, there were four protective genes $(\mathrm{HR}<1, \mathrm{P}<0.05)$ in the OTUD family, namely, OTUD1, OTUD2, OTUD6B, and OTUD7B (Fig. 1A). Then, least absolute shrinkage and selection operator (LASSO)-Cox regression analysis with 1000 replications was performed for these 4 prognostic genes in the TCGA-KIRC dataset and further showed that OTUD1 may be the key gene related to the overall survival (OS) of KIRC patients (Fig. 1B). Moreover, we showed that OTUD1 was not only downregulated in the KIRC tumor tissues but was also decreased along with increased KIRC tumor stage and grade (Fig. 1C, D, E). IHC analysis of OTUD1 in the KIRC tissue microarray (KIRC tumor $\mathrm{n}=38$, nontumor tissue $\mathrm{n}=38$ ) also showed that OTUD1 was downregulated in the tumor tissues ( $P$ $=0.004)$ (Fig. 1F, G). Finally, we revealed that low expression of OTUD1 was associated with shorter disease-free survival and OS times in ccRCC (Fig. $1 \mathrm{H}-\mathrm{J})$. Together, these data indicated that low expression of OTUD1 is correlated with a poor prognosis in ccRCC. 
A

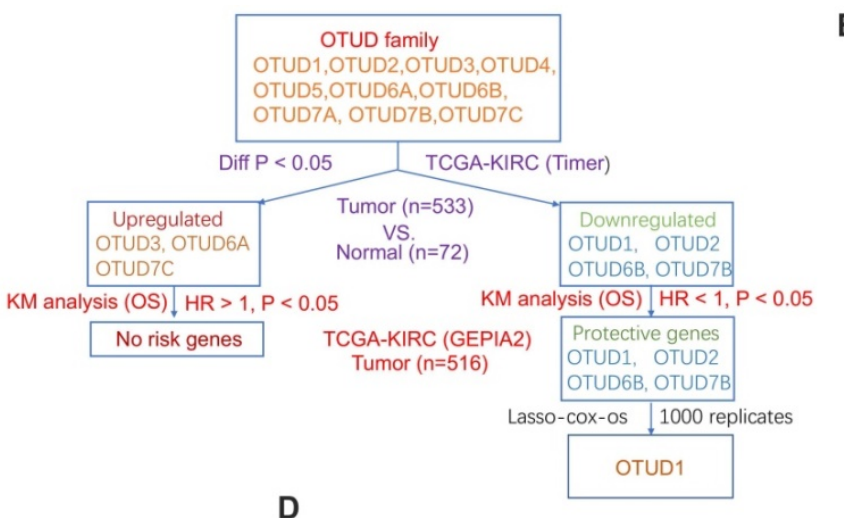

C

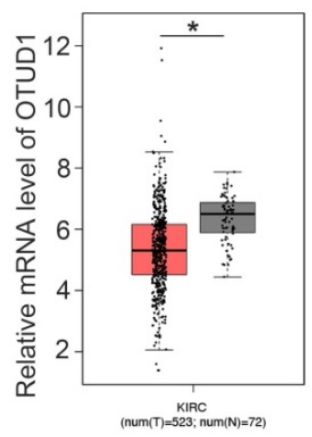

$\mathbf{F}$

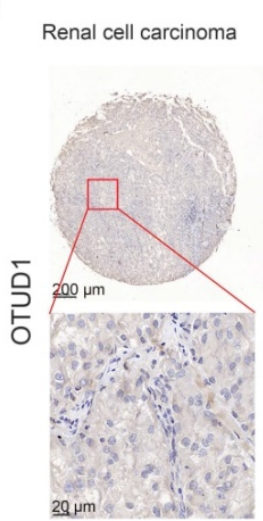

Adjacent non-tumor

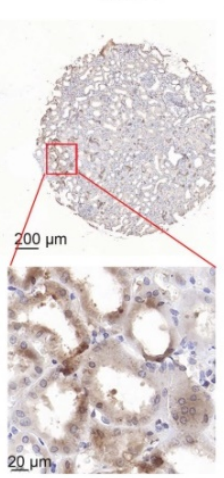

H

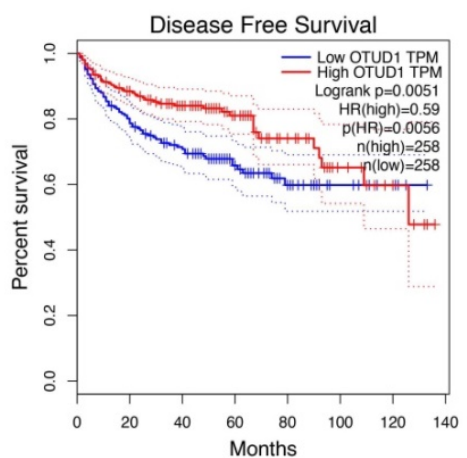
tissue

Expression of OTUD1 in KIRC based on individual cancer stages

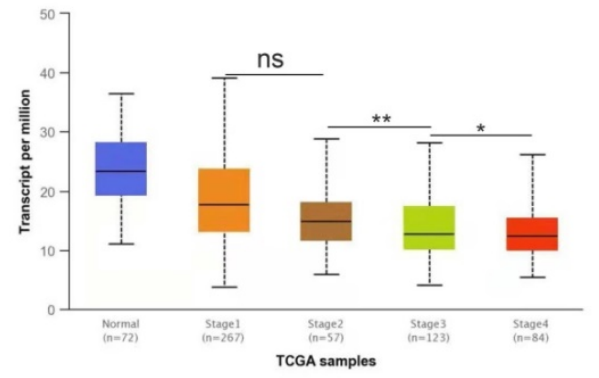

Adjacent non-tumor tissue

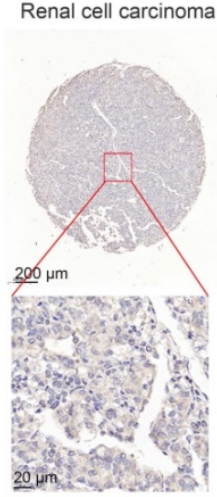

I

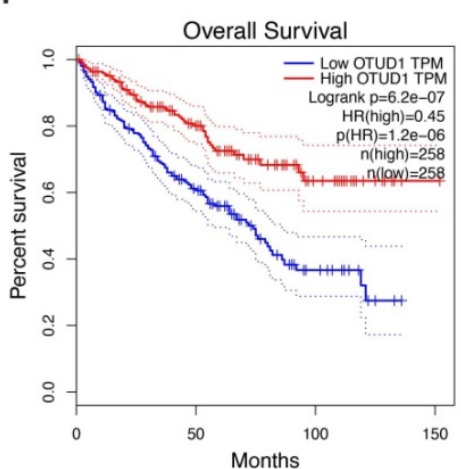

B

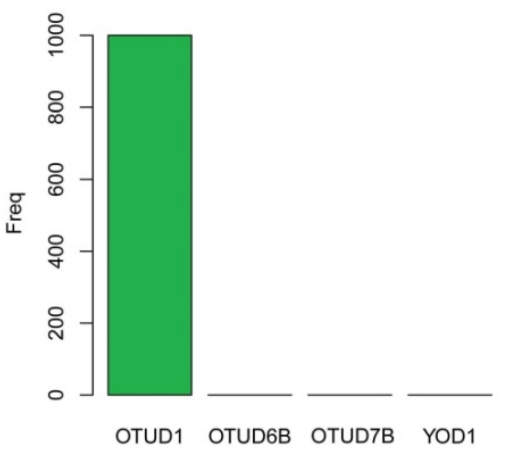

E

Expression of OTUD1 in KIRC based on tumor grade

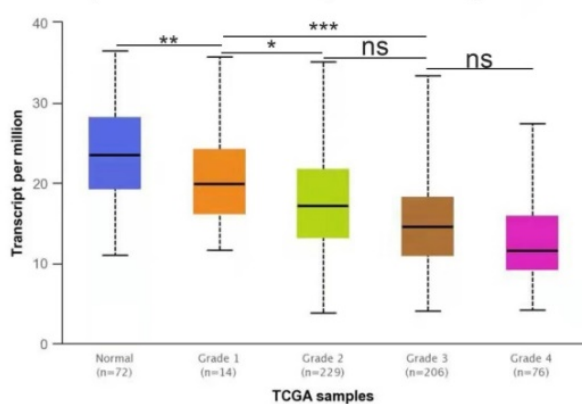

G

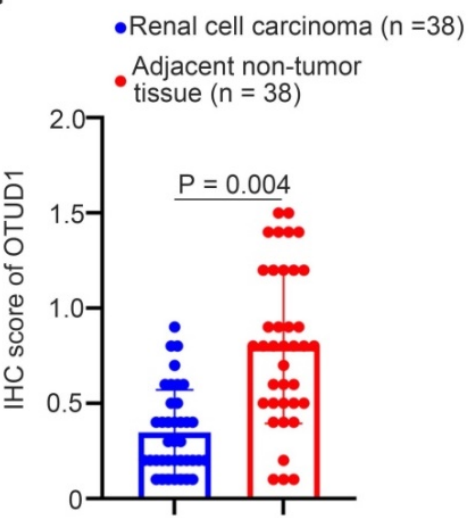

J

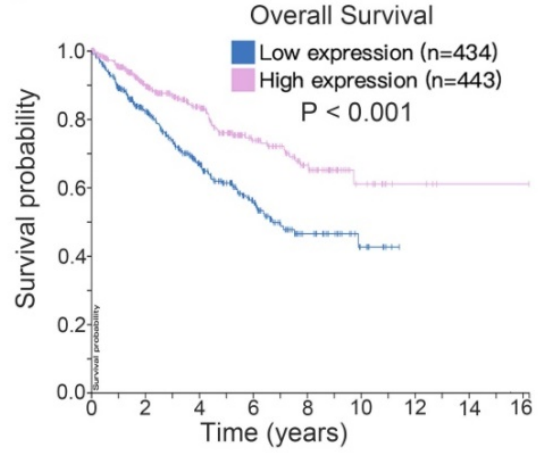

Figure 1. OTUDI is downregulated and correlated with an unfavorable prognosis in ccRCC patients. A, Identification of OTUDI from OTUD family in predicting prognosis of patients with KIRC. B, 10 -fold cross-validation with 1000 replications for variable selection in the LASSO-COX-OS model by minimum criteria (the 1 -SE criteria). C, differential expression analyses of OTUDI between tumor and normal tissues in TCGA-KIRC dataset. *, P $<0.05$. D, differential expression analyses of OTUDI in the different tumor stages of renal cancer in TCGA-KIRC. ns, not significant; *, $P<0.05$; **, $P<0.01$. E, differential expression analyses of OTUD1 in the different tumor grades of renal cancer in TCGA-KIRC. ns, not significant; *, P < 0.05; **, P $<0.01$. F and $\mathbf{G}$, IHC analysis of the tissue microarray by staining the OTUDI antibody. The typical image and expression level of OTUD1 in the non-tumor tissue and bladder cancer tissue were shown (G). P values as indicated. $\mathbf{H}-\mathrm{J}$, Kaplan-Meier analysis with two-sided log-rank test was conducted using GEPIA2 or The human protein atlas to evaluate the differences in Disease-free survival $(\mathrm{H})$ and overall survival $(\mathrm{I}$ and $\mathrm{J})$ time between the patients with high and low expression of OTUD1 in TCGA-KIRC dataset. 


\section{OTUD1 loss promotes cell proliferation and regulates the cell cycle of renal cancer cells}

Then, we studied the biological role of OTUD1 in renal cancer cells. The expression level of OTUD1 was downregulated after transfection with OTUD1specific siRNAs (Fig. 2A, 2B). The cell proliferation activity of these cells was measured by Cell Counting Kit-8 (CCK-8) assay (Fig. 2C). We showed that knockdown of OTUD1 promoted cancer cell growth in both 786-O and ACHN cells (Fig. 2C). Intriguingly, cell cycle analysis indicated that OTUD1 silencing induced G1-G2 phase transition in 786-O cells (Fig. 2D, 2E). In contrast, the HA-OTUD1 constructs were transfected into 786-O and ACHN cells to ectopically overexpress OTUD1 (Fig. 2F, 2G). The CCK-8 assay showed that overexpression of OTUD1 decreased the proliferation of renal cancer cells (Fig. 2H). Moreover, a cell cycle assay indicated that overexpression of OTUD1 blocked the G1 to G2 phase transition in 786-O cells (Fig. 2I, 2J). Given that OTUD1 functioned as a deubiquitinating enzyme, we would like to investigate whether OTUD1 inhibit the proliferation of renal cancer cells through its deubiquitinating activity. The inactive mutant of OTUD1 (OTUD1 C320S) [17, 18] and OTUD1 wild type (WT) were transfected into the 786-O and ACHN cells, respectively (Fig. 2K). It was not surprising that overexpression of inactive mutant of OTUD1 made little effect on the renal cancer cells proliferation compared to the OTUD1 wild type (Fig. 2L). Furthermore, the nude mouse studies showed that OTUD1 depletion led to tumor growth in vivo (Fig. $2 \mathrm{M}-2 \mathrm{O})$. Together, these results suggest that OTUD1 contributes to inhibiting renal cancer cell proliferation.

\section{OTUD1 is a negative regulator of the PI3K/AKT and TNF-alpha/NF-kappa B signaling pathways in ccRCC}

To identify the underlying mechanism by which OTUD1 inhibits ccRCC tumor growth, transcriptome sequencing after OTUD1 ablation was performed in ACHN cells (Fig. 3A, B). Kyoto Encyclopedia of Genes and Genomes (KEGG) enrichment pathway analysis showed that knockdown of OTUD1 activated a number of cellular processes and signaling pathways with $\mathrm{P}$ values less than 0.01 (Fig. 3C). Among them, the PI3K-AKT, TNF and NF-kappa B signaling pathways were the most altered signaling pathways after OTUD1 silencing (Fig. 3B). Gene set enrichment analysis (GSEA) of the RNA-seq dataset showed that AKT signaling was activated in the siOTUD1 group (Fig. 3C). We also found that knockdown of OTUD1 increased the phosphorylation levels of AKT, but overexpression of OTUD1 decreased the phospho- rylation levels of AKT in both 786-O and ACHN cells (Fig. 3D, E). Moreover, GSEA of the RNA-seq dataset demonstrated that OTUD1 silencing resulted in the hyperactivation of TNF and the NF-kappa B signaling pathway (Fig. 3F, G). Consistently, proteomics analysis also indicated that OTUD1 was closely associated with the inhibition NF-kappa B signaling pathway in renal cell carcinoma (Fig. 3H). Furthermore, we found that OTUD1 knockdown increased the expression of downstream target genes of TNF and the NF-kappa B signaling pathway, such as TNF, BIRC3, CXCL3, GADD45a, GADD45b, and CXCL8, in 786-O and ACHN cells (Fig. 3I, J). In contrast, ectopic overexpression of OTUD1 reduced the expression of these genes in 786-O cells (Fig. 3K). NF-kappa B signaling is essential for maintaining ccRCC cell survival [19]. We investigated whether OTUD1 inhibits the tumor growth of ccRCC by inactivating NF-kappa B. We treated 786-O cells with two distinct NF-kappa B inhibitors (IKK-16 and JSH-23) after silencing OTUD1 (Fig. 3L, M) and found that NF-kappa B inhibitors diminished the growth-promoting effect induced by the knockdown of OTUD1 (Fig. 3L, M). Thus, these data suggest that OTUD1 participates in inactivating the AKT and NF-kappa B/TNF-alpha signaling pathways in ccRCC.

\section{OTUD1 is responsible for stabilizing PTEN in CCRCC}

Since OTUD1 is reported to be a deubiquitinase and involved in protein posttranslational modification, we explored the potential binding partners of OTUD1 by employing bioinformatic analysis. OTUD1 was responsible for repressing the PI3K/AKT signaling pathway and NF-kappa B signaling pathway in renal cancer cells (Fig. 3). And PI3K/AKT pathway is also reported to activate NF-kappa B signaling pathway in cells $[13,20]$. We downloaded the dataset from the UbiBrowser and analyzed the interaction scores of AKT pathway related proteins with OTUD1. The data was indicated in Table S3. We found that OTUD1 might interact with PTEN, AKT1, AKT2, AKT3, and mTOR among these related proteins (Fig. 4A). Given that OTUD1 promotes the activation of AKT and the NF-kappa B/TNF-alpha signaling pathway in ccRCC, OTUD1 can usually stabilize its substrates through its deubiquitinase activity. Thus, we investigated whether OTUD1 binds with and stabilized PTEN, which is a well-known negative regulator of the AKT and NF-kappa B/TNF-alpha signaling pathways [13]. First, the reciprocal immunoprecipitation assay indicated that endogenous OTUD1 interacts with PTEN in 786-O and ACHN cells (Fig. 4B, C). 
Meanwhile, we also found that AKT did not bind with OTUD1 in 786-O cells (Supplementary Fig. 1A). Knockdown of OTUD1 decreased PTEN expression, and this process was suppressed by $26 \mathrm{~S}$ proteasome inhibitor (MG132) treatment in 786-O cells (Fig. 4D). In contrast, overexpression of OTUD1 WT but not the catalytic inactive mutant of OTUD1 (OTUD1 C320S) led to an increase in PTEN expression in 786-O cells (Fig. 4E). Moreover, we showed that OTUD1 silencing promoted the polyubiquitination of PTEN, but OTUD1 WT overexpression suppressed the polyubiquitination of PTEN in 786-O cells (Fig. 4F, G). Notably, we also showed that the OTUD1 C320S mutant made little effect on the change of polyubiquitination of PTEN compared to OTUD1 WT (Fig. 4G). It is known that OTUD1 efficiently degrades the K63-linked ubiquitin chain [21], which does not contribute to proteasome degradation. While, Zhengkui Zhang et al. reported that OTUD1 regulates the K48-linked ubiquitination of SMAD7 to stabilize SMAD7 in breast cancer [22]. We then demonstrated that OTUD1 regulates the K48-linked ubiquitination of PTEN, which suggested that OTUD1 might prevent PTEN proteasomal degradation in renal cancer cells (Fig. $4 \mathrm{H}$ ). In addition, IHC was performed on the KIRC tissue microarray by using the PTEN antibody (Fig. 4I). We found that there was a positive correlation between OTUD1 and PTEN in KIRC tissue samples (Spearman correlation $r=0.4798, \mathrm{P}=0.0023$ ) (Fig. 4J). Therefore, OTUD1 might be the deubiquitinase of PTEN in ccRCC.

Next, we wondered whether OTUD1 inhibits cancer cell growth through PTEN in ccRCC cells. 786-O cells were transfected with siNC, siPTEN, siOTUD1, or siPTEN plus siOTUD1 (Fig. 4K). We found that cotransfection with siPTEN and siOTUD1 attenuated the increase in phosphorylated AKT, VCAM1 and CXCL8 and cancer cell proliferation induced by knockdown of OTUD1 alone (Fig. 4K-M). We also showed that PTEN ablation could attenuate the decrease in phosphorylated AKT, VCAM1 and CXCL8 and cancer cell proliferation mediated by overexpression of OTUD1 in 786-O cells (Fig. 4N-P). Together, our data suggest that OTUD1 deubiquitinates and stabilizes PTEN in ccRCC.

\section{OTUDI contributes to regulating the sensitivity of renal cancer cells to TKIs}

PTEN is responsible for influencing the sensitivity of cancer cells to multiple types of small molecules, such as AKT inhibitors, MEK inhibitors, and TKIs [23-25]. According to the above findings, we aimed to determine whether OTUD1 regulates the sensitivity of renal cancer cells to antitumor regimens. First, we revealed that the half-maximal inhibitory concentration (IC50) values of sunitinib in the OTUD1 overexpression group were lower than those in the control group in 786-O cells (Fig. 5A). Currently, TKIs are the first-line targeted treatment for ccRCC [26]. The acquired resistance to TKIs in ccRCC has become the major obstacle for improving the survival of ccRCC patients [27]. Thus, we aimed to study the specific role of OTUD1 in regulating the sensitivity of renal cancer cells to TKIs. We showed that OTUD1 WT overexpression decreased the IC50 values of sunitinib in both 786-O and ACHN cells (Fig. 5B), but ectopically overexpressed the OTUD1 C320S mutant made little effect on the IC50 values of sunitinib compared to control group (empty vector) in renal cancer cells (Supplementary fig. 1B). Overexpression of OTUD1 increased apoptosis after treatment with sunitinib versus that in the control group in both 786-O and ACHN cells (Fig. 5C-F). In contrast, we showed that OTUD1 depletion decreased the apoptosis of renal cancer cells treated with sunitinib (Fig. 5G-J) and increased the IC50 values of sunitinib in 786-O and ACHN cells (Fig. 5K). Moreover, the in vivo study also demonstrated that OTUD1 ablation reduced the sensitivity to sunitinib (Fig. 5L-N). Together, these data suggest that OTUD1 participates in modulating the sensitivity of renal cancer cells to TKIs.

\section{OTUDI sensitizes ccRCC cells to the TKIs via PTEN}

Next, we investigated whether OTUD1 regulates the sensitivity of renal cancer cells to TKIs through PTEN. It was not surprising that PTEN silencing diminished the change in IC50 values of sunitinib, AKT phosphorylation, VCAM-1, CXCL8 and cleaved caspase 3 levels induced by silencing or overexpressing OTUD1 in ccRCC cells (Fig. 6A-F). We previously analyzed a TKI resistance dataset (GSE76068) from a mouse model and showed that RRM2 is closely associated with sunitinib resistance in renal cancer, and this effect is mediated by its activation of the AKT signaling pathway [28]. It has been also documented that the NF-kappa B signaling pathway was involved in modulating the sensitivity of TKIs in renal cancer cells [29]. In consist with previously finding, we reanalyzed the TKI resistance dataset (GSE76068) and performed KEGG pathway enrichment analysis to show that TNF and NF-kappa B signaling pathways were correlated with TKI resistance (Supplementary fig. 1C-E). Moreover, we showed that inhibition of the TNF/NF-kappa B signaling pathway enhanced the antitumor effect of TKIs in both 786-O and ACHN cells (Supplementary fig. 1F-I). 

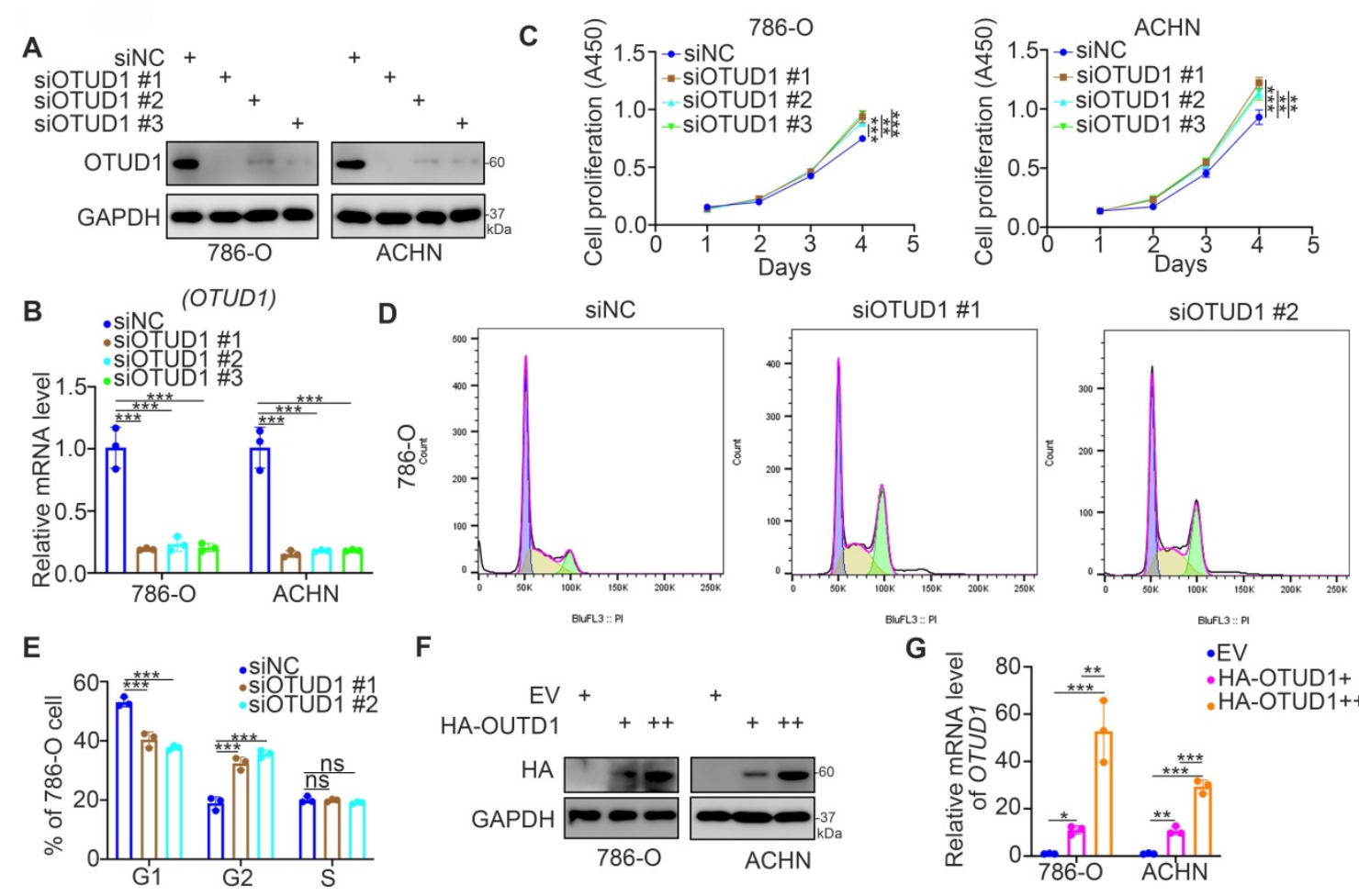

$\mathbf{F}$
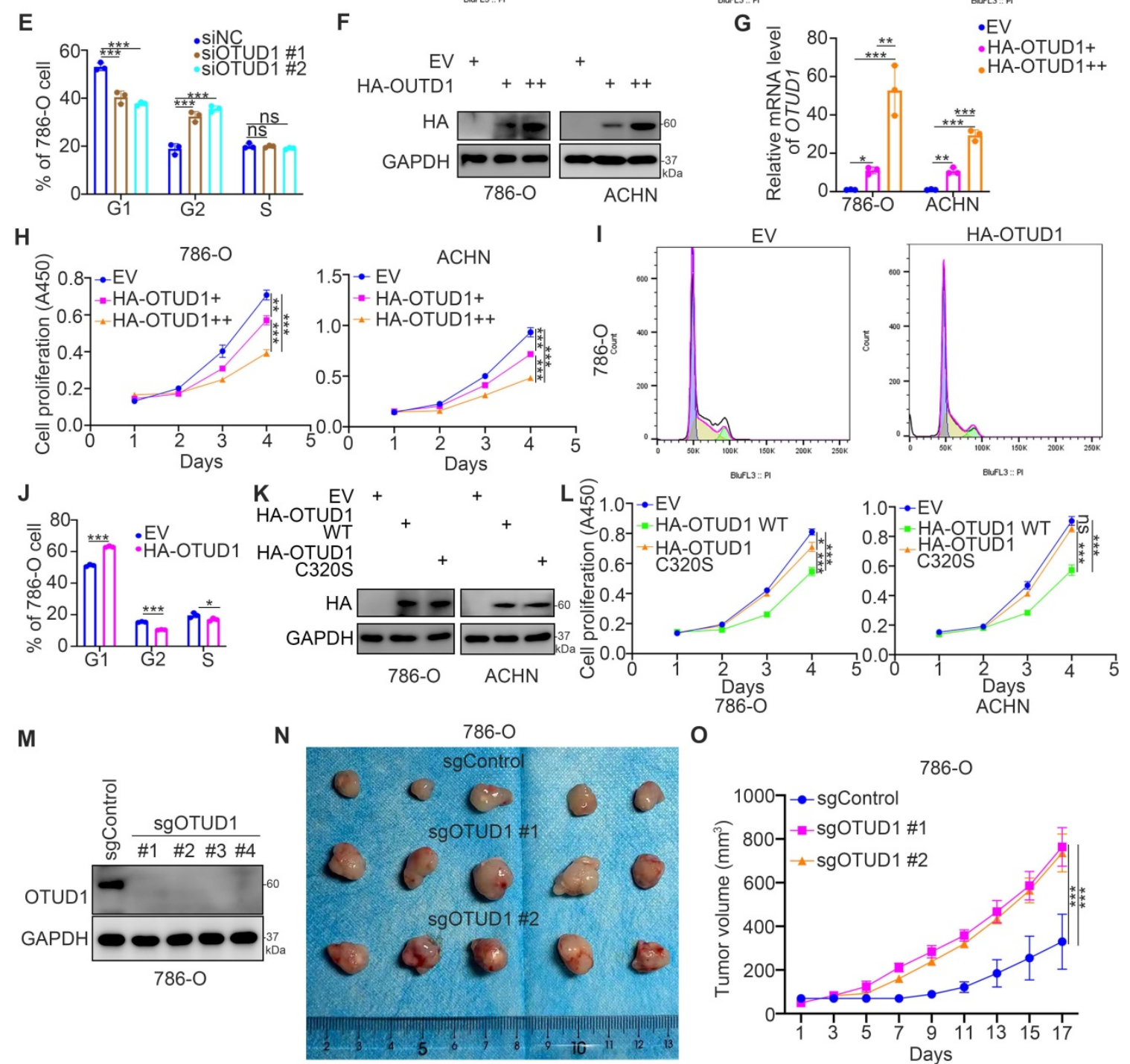

0

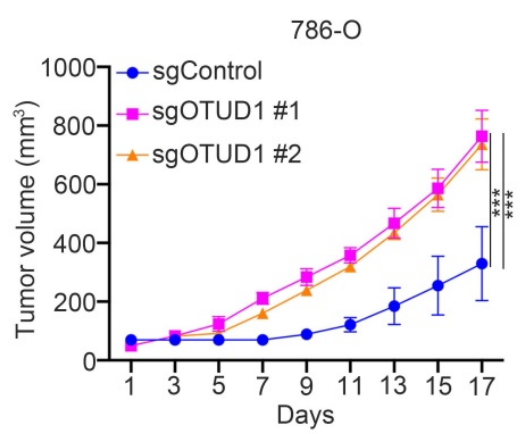

Figure 2. OTUDI loss promotes cell proliferation and regulates the cell cycle of renal cancer cells. A-E, 786-O and ACHN cells were transfected with indicated siRNAs for $48 \mathrm{~h}$. Cells were collected for Western blot analysis (A), RT-qPCR analysis (B), CCK-8 assay (C), cell cycle assay (D and E). For panel B and C, data presents as mean \pm SD with three replicates. **, $\mathrm{P}<0.01$; ***, $\mathrm{P}<0.001$. For panel $\mathrm{E}$, data presents as mean $\pm \mathrm{SD}$ with two replicates. Ns, not significant; **, $\mathrm{P}<0.01$; ***, $\mathrm{P}<0.001$. F-J, 786-O and ACHN cells were transfected with indicated empty vector, HA-OTUDI (2ng), or HA-OTUDI (4ng) for $48 \mathrm{~h}$. Cells were harvested for Western blot analysis (F), RT-qPCR analysis $(\mathrm{G})$, CCK-8 assay $(\mathrm{H})$, cell cycle assay $(\mathrm{I}$ and J). For panel $\mathrm{G}$ and $\mathrm{H}$, data presents as mean \pm SD with three replicates. **, $\mathrm{P}<0.01$; ***, $\mathrm{P}<0.001$. For panel J, data presents as mean \pm SD with two replicates. Ns, not significant; **, $\mathrm{P}<0.01 ; * * *, \mathrm{P}<0.001$. K and L, 786-O and ACHN cells were transfected with indicated empty vector for $48 \mathrm{~h}$. Cells were harvested for Western blot analysis (K) and CCK-8 assay (L). Data presents as mean \pm SD with three replicates. Ns, not significant; *, P< $0.05 ; * * *, P<0.001$. M-O, 786-O cells were infected with indicated sgRNAs. After puromycin selection, cells were harvested for Western blot analysis (M). Then, cells were subcutaneously injected into the flank of nude mice. The tumor image was shown in panel $\mathrm{N}$. The tumor growth curve was shown in panel $\mathrm{O}$. Data presents as mean \pm SD with five replicates. $* * * *, \mathrm{P}<0.001$. 
A

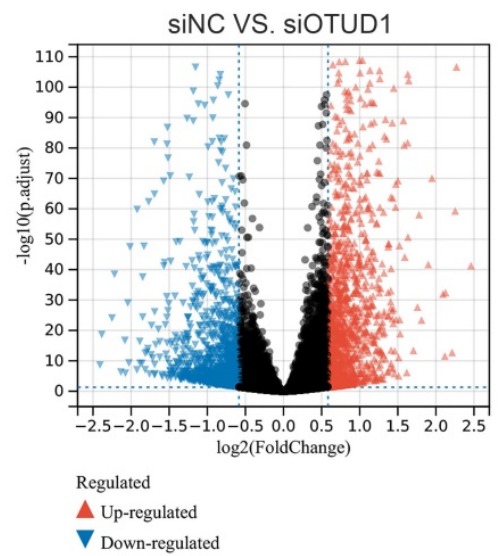

C

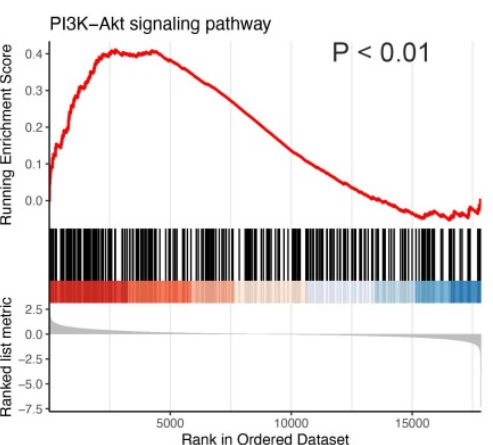

$\mathbf{F}$

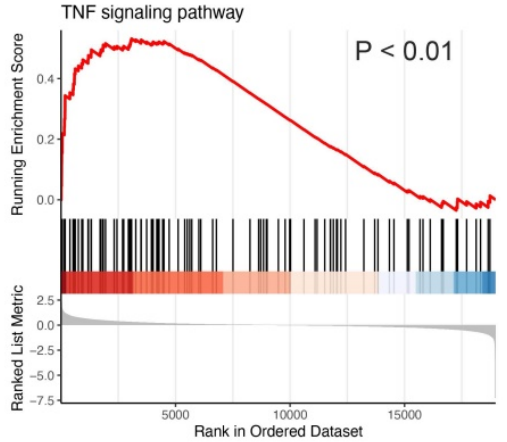

786-O

I. $\quad \cdot \operatorname{sinC}$

- siNC 1 TOD1 \#1

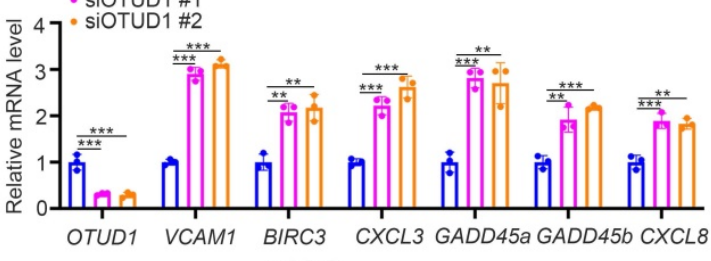

$786-0$

K

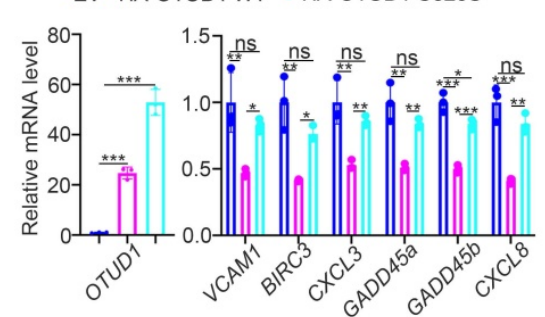

B

D
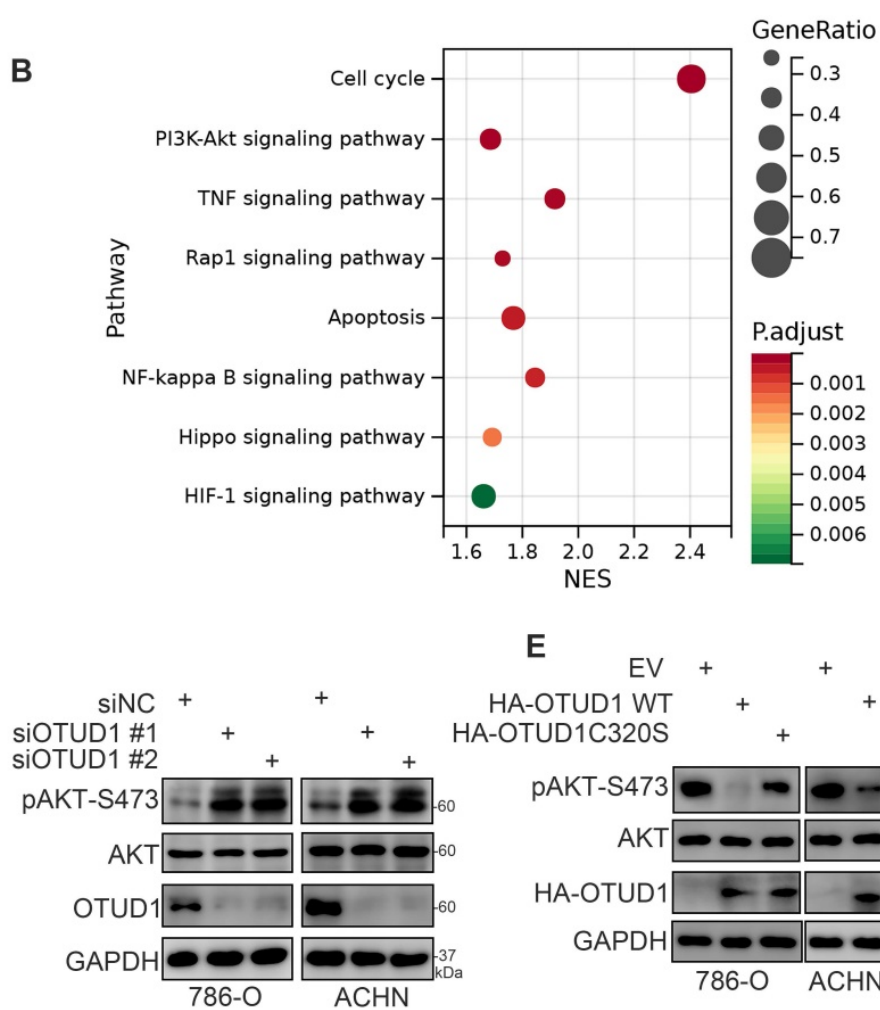

E

$\underset{\text { HA-OTUDD1C320S }}{\text { EV }}{ }^{+}+{ }^{+}+{ }^{+}+$

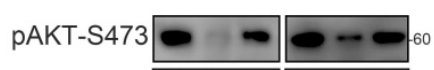

AKT $-2-0-60$

HA-OTUD1 -

$\mathrm{GAPDH}-\boldsymbol{- \infty}-\infty \square_{\mathrm{KDa}}^{37}$

786-O ACHN

H
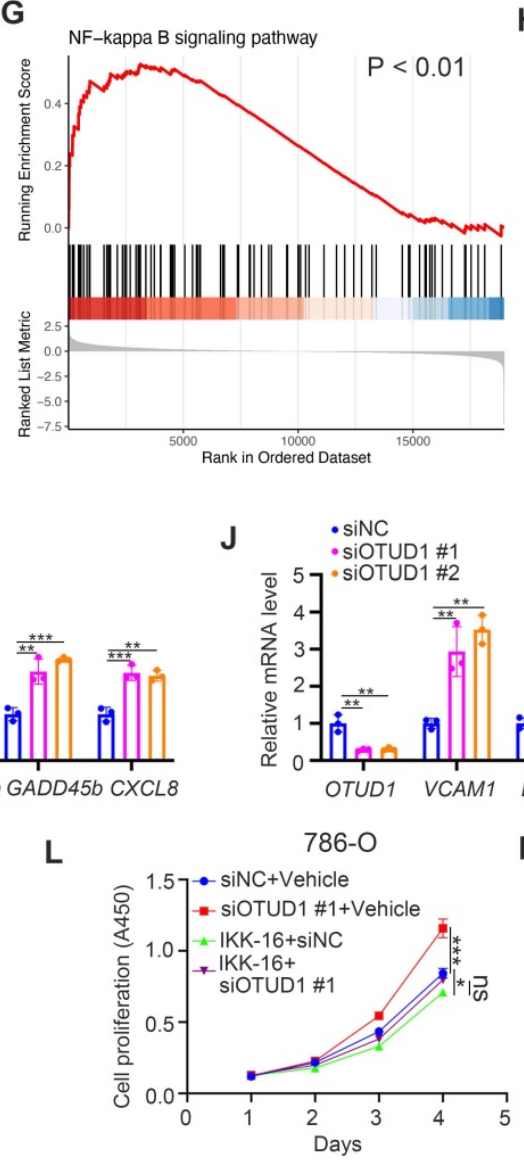

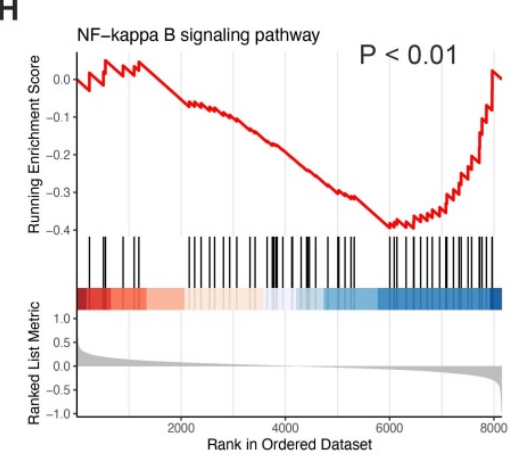

ACHN
L
M

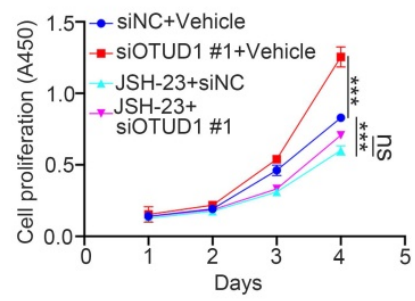

Figure 3. OTUDI is a negative regulator of the PI3K/AKT and TNF-alpha/NF-kappa B signaling pathways in ccRCC. A, 786-O cell was transfected with siNC or siOTUDI for $48 \mathrm{~h}$. These cells were subjected to RNA-seq analysis. The volcano plot showed the genes changed after knockdown of OTUDI (A). B, KEGG pathway enrichment analysis indicated that the pathways were changed after knockdown of OTUD1 in 786-O cells. C, GSEA analysis showed PI3K-AKT pathway was activated by knocking down OTUD1 in 786-O cells. P values as indicated. D, 786-O and ACHN cells were transfected with indicated siRNAs for $48 \mathrm{~h}$. Cells were collected for Western blot analysis. E, 786-O and ACHN cells were transfected with indicated plasmids for $48 \mathrm{~h}$. Cells were collected for Western blot analysis. F and G, GSEA analysis showed TNF signaling pathway 
(F) and NF-kappa B signaling (G) were activated by knocking down OTUDI in 786-O cells. P values as indicated. H, NF-kappa B pathway was down-regulated in patients with high OTUDI compared with patients with low OTUDI expression in TCGA database. I and J, 786-O and ACHN cells were transfected with indicated siRNAs for $48 \mathrm{~h}$. Cells were harvested for RT-qPCR analysis. Data presents as mean \pm SD with three replicates. $*, P<0,05 ; * *, P<0.01$; ***, $P<0.001 . \mathbf{K}, 786-O$ cells were transfected with indicated plasmids for $48 \mathrm{~h}$. Cells were harvested for RT-qPCR analysis. Data presents as mean \pm SD with three replicates. *, $\mathrm{P}<0,05 ; * *, \mathrm{P}<0.01$; ***, $\mathrm{P}<0.001$. $\mathbf{L}$ and $\mathbf{M}, 786-\mathrm{O}$ cells were transfected with indicated siRNAs for $24 \mathrm{~h}$. These cells were treated with or without $500 \mathrm{nM} \mathrm{IKK-16} \mathrm{(L)} \mathrm{or} 10 \mu \mathrm{M}$ JSH-23 (M) and subjected to CCK-8 assay. Data presents as mean \pm SD with three replicates. Ns, not significant; $*, P<0,05 ; * * *, P<0.001$.

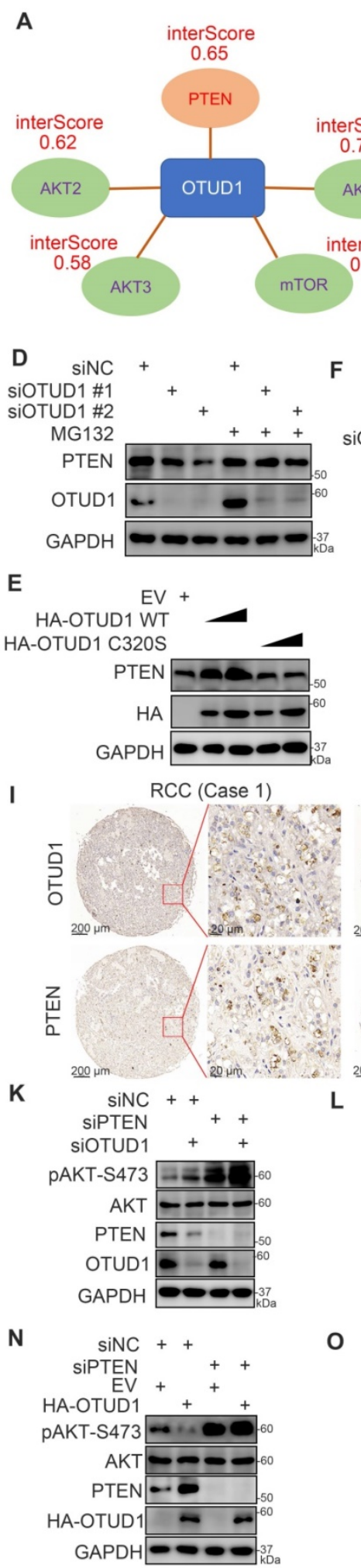

B
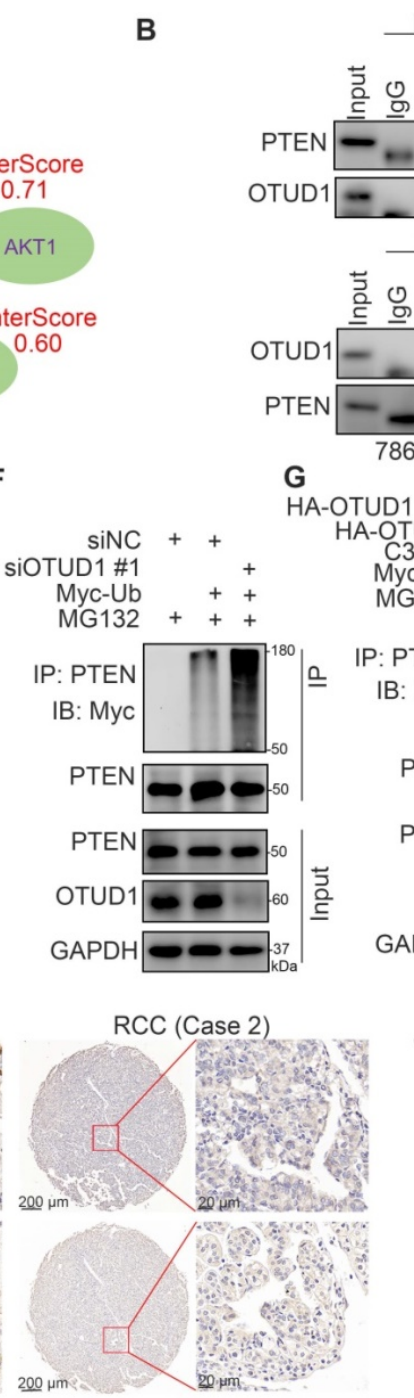

L
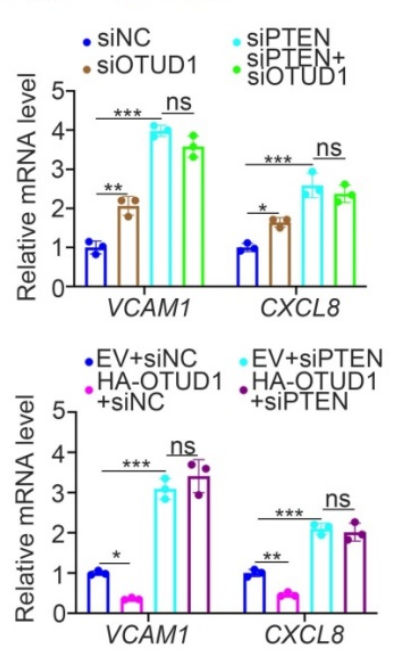

$\mathbf{J}$
C

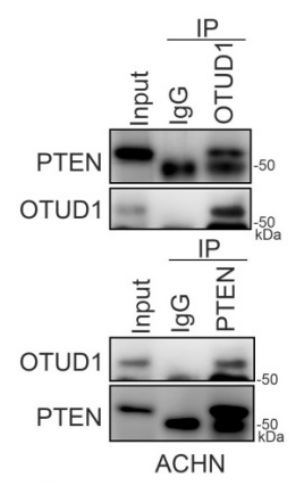
H Myc-Ub WT K48 K63
HA-OTUD1 $+{ }^{+}+{ }^{+}+$
MG132 + + + + +

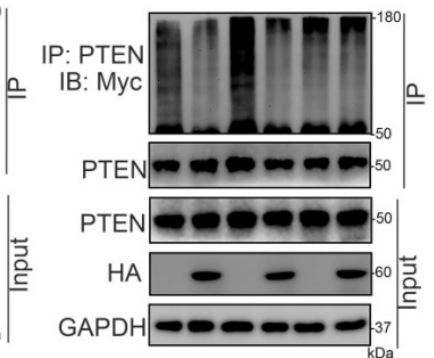

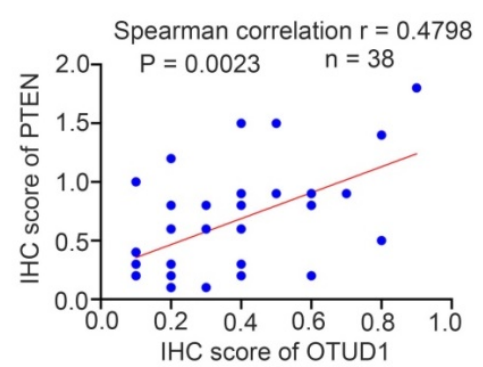

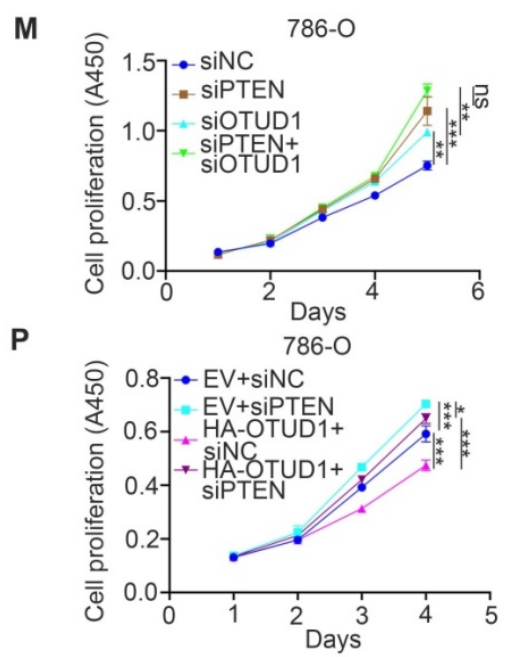

Figure 4. OTUD1 is responsible for stabilizing PTEN in ccRCC. A, We downloaded the dataset from the UbiBrowser and analyzed the interaction scores of AKT pathway related proteins with OTUD1. The interaction scores (interScore) of PTEN, AKT1, AKT2, AKT3 and mTOR were indicated in panel A. B, The cell lysate of 786-O cells were collected and incubated with OTUDI or PTEN antibodies for immunoprecipitation assay. C. The cell lysate of ACHN cells were collected and incubated with OTUDI or PTEN antibodies for immunoprecipitation assay. D, 786-O cells were transfected with indicated siRNAs for $48 \mathrm{~h}$. Before harvesting cells for Western blot analysis, cells were 
treated with or without MG132 for 8 h. E, 786-O cells were transfected with indicated plasmids for 48 h. Cells were collected for Western blot analysis. F, 786-O cells were transfected with indicated constructs for $48 \mathrm{~h}$. Before harvesting cells for immunoprecipitation by using the PTEN antibody, cells were treated with or without MG 132 for $8 \mathrm{~h}$. G, 786-O cells were transfected with indicated constructs for $48 \mathrm{~h}$. Before harvesting cells for immunoprecipitation by using the PTEN antibody, cells were treated with or without MG132 for $8 \mathrm{~h}$. H, 786-O cells were transfected with indicated constructs for $48 \mathrm{~h}$. Before harvesting cells for immunoprecipitation by using the PTEN antibody, cells were treated with or without MG132 for $8 \mathrm{~h}$. I and J, IHC analysis of the tissue microarray by staining the OTUDI and PTEN antibodies. The correlation between these two proteins was shown in panel I, P values as indicated. K-M, 786-O cells were transfected with indicated siRNAs for $48 \mathrm{~h}$. Cells were harvested for Western blot analysis (J), RT-qPCR analysis $(\mathrm{K})$, and CCK-8 assay $(\mathrm{L})$. For panel $\mathrm{K}$ and $\mathrm{L}$, data presents as mean \pm SD with three replicates. *, $\mathrm{P}<0,05 ; * *, P<0.01$; ***, $\mathrm{P}<0.001 . \mathbf{N}-\mathrm{P}, 786-\mathrm{O}$ cells were transfected with indicated plasmids for $48 \mathrm{~h}$. Cells were harvested for Western blot analysis $(\mathrm{M}), \mathrm{RT}-\mathrm{qPCR}$ analysis $(\mathrm{N})$, and CCK-8 assay $(\mathrm{O})$. For panel $\mathrm{N}$ and $\mathrm{O}$, data presents as mean \pm SD with three replicates. *, $\mathrm{P}<0,05$; **, $\mathrm{P}<0.01$; ***, $\mathrm{P}<0.001$.

A

C
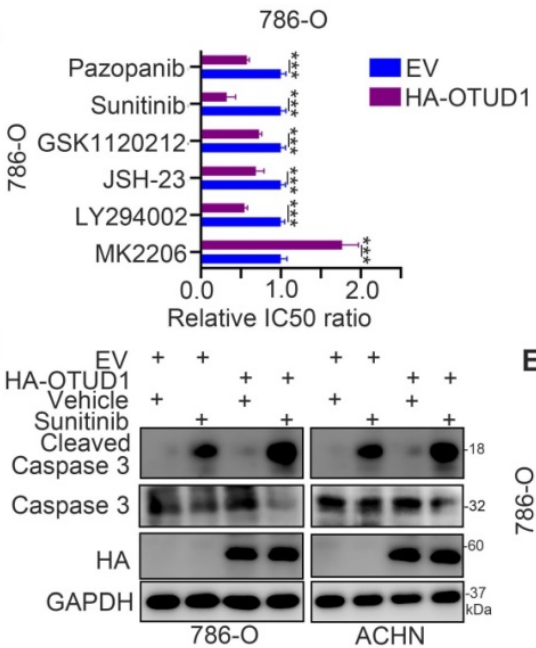

D

:EV+Vehicle : :HA-OTUD1+Vehicle

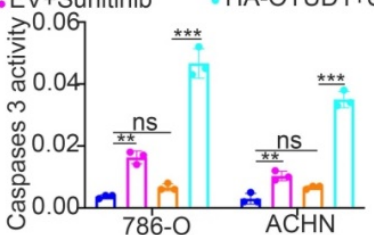

$\mathrm{H}$ -sgControl+Vehicle $\quad$ sgOTUD1+Vehicle
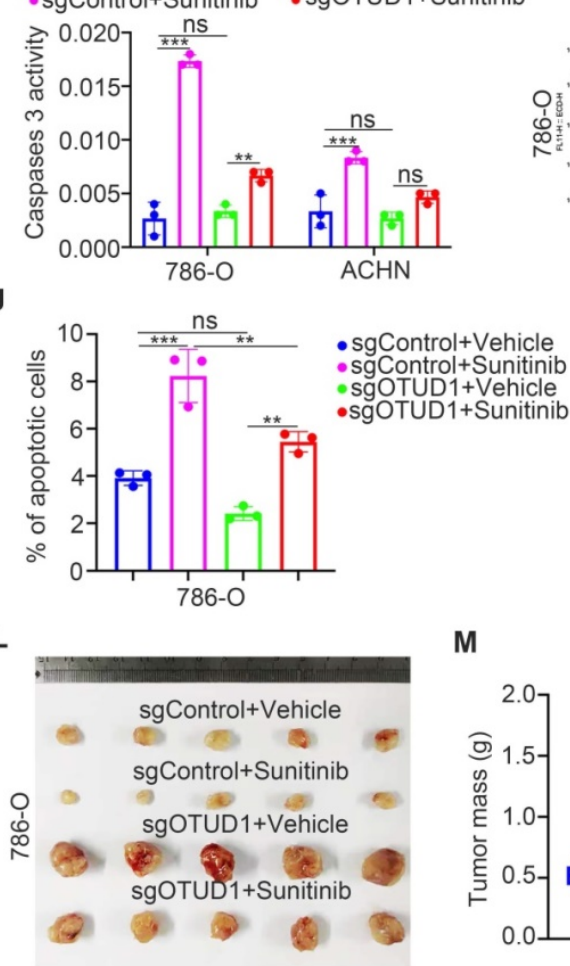

E

M
B
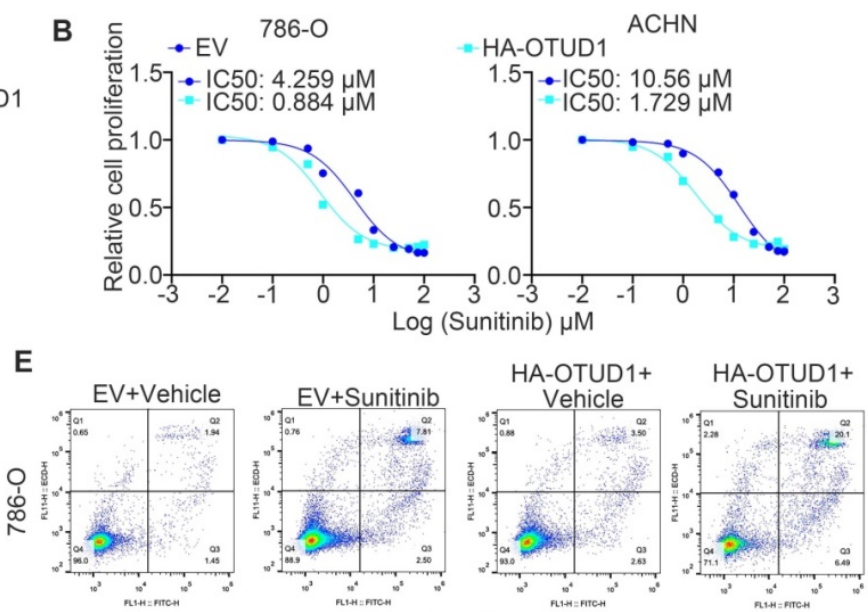

annexin v-fitc/pi

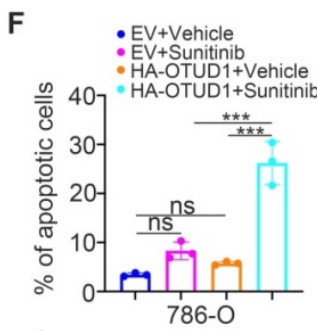

G
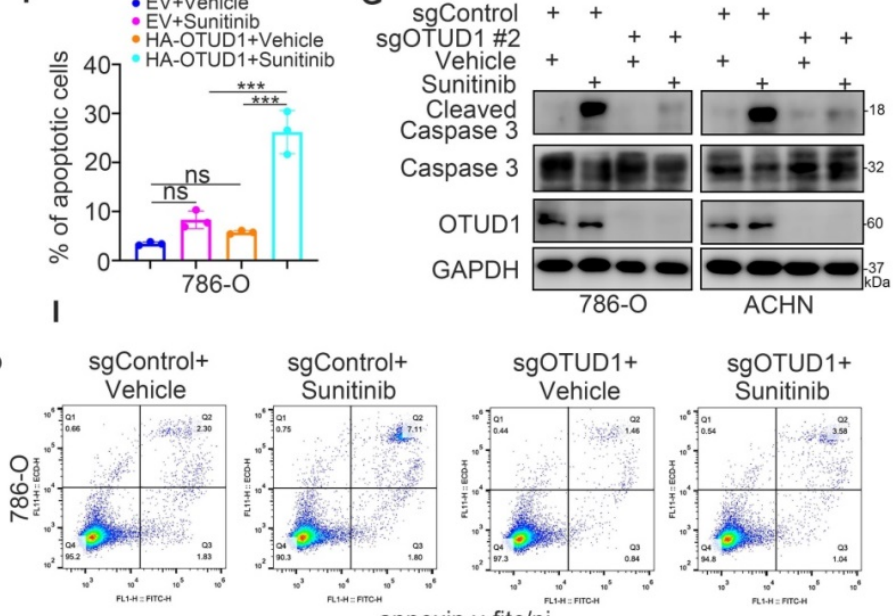

annexin $v$-fitc/pi

K
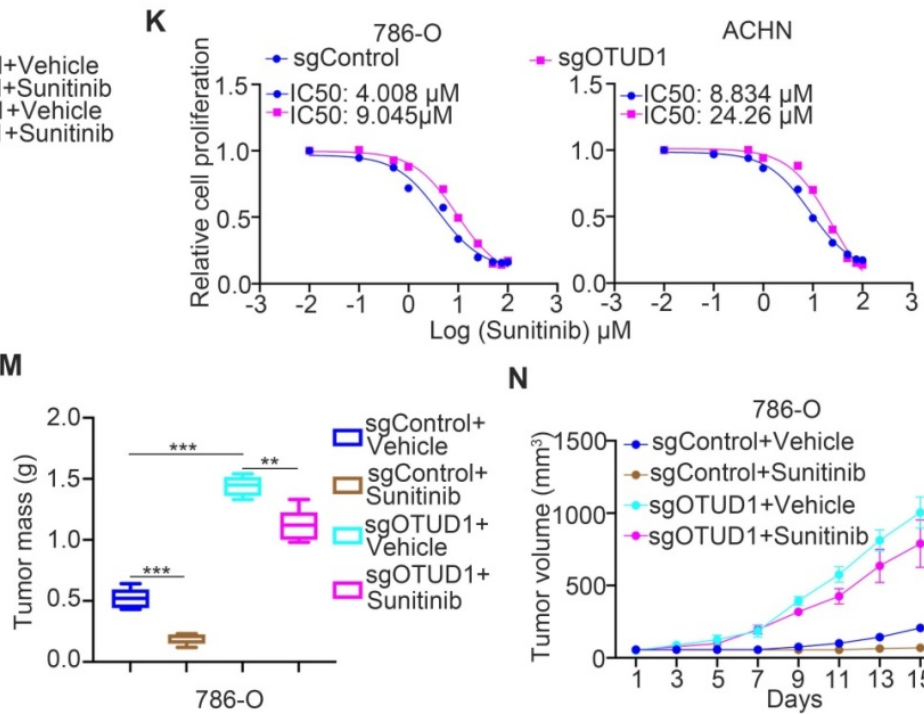

N

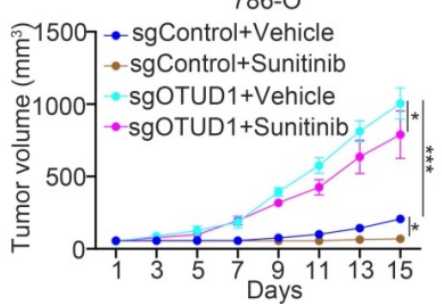

Figure 5. OTUDI contributes to regulating the sensitivity of renal cancer cells to TKIs. A, 786-O cells were transfected indicated plasmids for $48 \mathrm{~h}$. These cells were treated with a serial dose of small molecules indicated in the figure and measure the IC50 values of each small molecules. The EV/HA-OTUDI IC50 ratios of each small 
molecules were shown in the panel A. Data presents as mean \pm SD with three replicates. ***, $\mathrm{P}<0.001$. B, 786-O and ACHN cells were transfected with indicated plasmids for $48 \mathrm{~h}$. Cells were treated with a serial concentration of Sunitinib for measuring the IC50 values of Sunitinib. C-F, 786-O and ACHN cells were transfected with indicated constructs for $48 \mathrm{~h}$. Cells were treated with or without Sunitinib and collected for Western blot analysis (C), Caspase 3 activity assay (D), and Annexin v-FITC/PI assay (E and F). For panel D, E, and F, data presents as mean \pm SD with three replicates. Ns, not significant; **, $P<0.01$; ***, $P<0.001$. G-J, 786-O and ACHN cells were infected with indicated constructs for $72 \mathrm{~h}$. After puromycin selection, cells were treated with or without Sunitinib and collected for Western blot analysis $(\mathrm{G})$, Caspase 3 activity assay $(\mathrm{H})$, and Annexin v-FITC/PI assay (I and J). For panel H, I, and J, data presents as mean \pm SD with three replicates. Ns, not significant; **, P<0.01; ***, P<0.001. K, 786-O and ACHN cells were infected with indicated constructs for $72 \mathrm{~h}$. After puromycin selection, cells were treated a serial concentration of Sunitinib for measuring the IC50 values of Sunitinib. L-N, 786-O cells were infected with indicated constructs for $72 \mathrm{~h}$. After puromycin selection, cells were subcutaneously injected into the flank of nude mice. Mice were treated with or without Sunitinib. The tumor image was shown in panel L. The tumor mass was shown in panel M. The tumor-growth curve was shown in panel N. Data presents as mean \pm SD with five replicates. *, $\mathrm{P}<0.05 ; * *, \mathrm{P}<0.01 ; * * *, \mathrm{P}<0.001$.

A

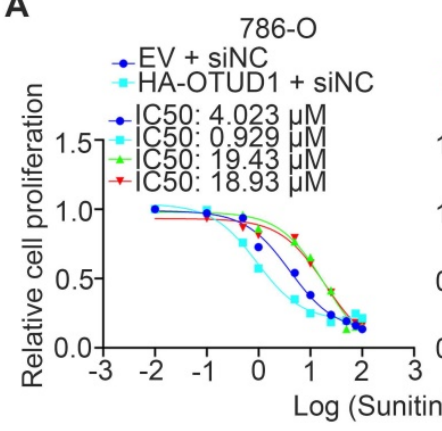

C

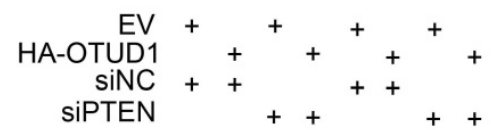

Sunitinib $(1 \mu \mathrm{M})++++++++$

Caspase $3-2=-2$ cleavedpAKT-S473

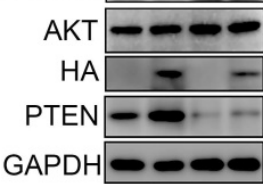

786-O

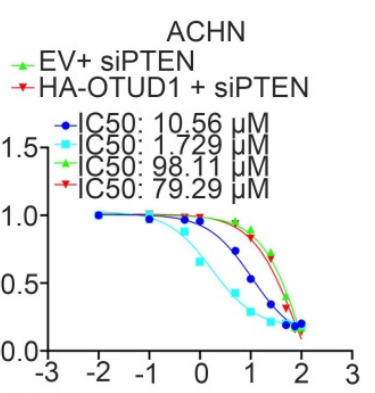

$\sum_{\text {II }} \cdot E V+\operatorname{sinC}$

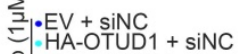

- HA-OTUD1 + siNC

:EV+ SiPTEN

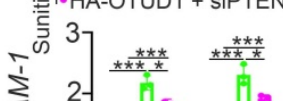

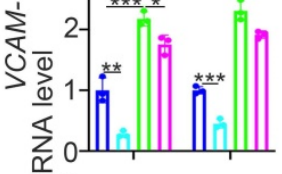

.

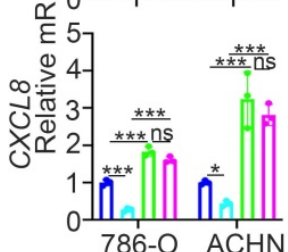

B

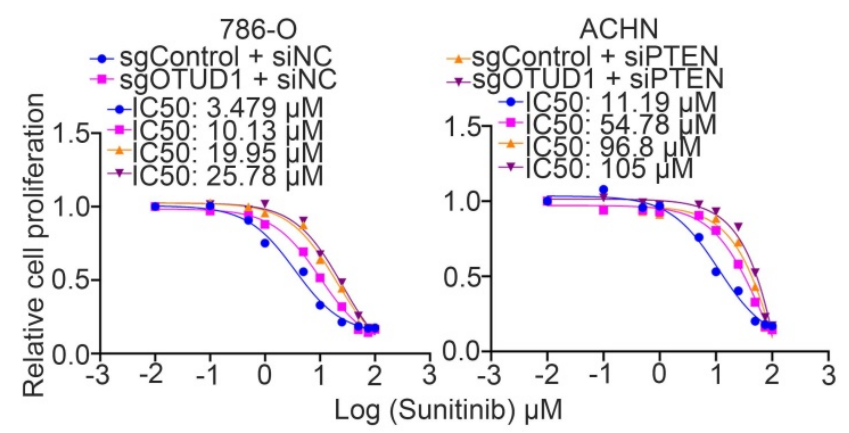

-sgControl + siNC

E

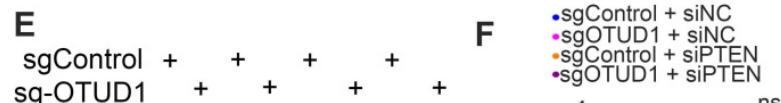

sg-OTUD1 $+\quad+\quad+\quad+$ $\underset{\text { SiPTEN }}{\operatorname{sinC}}++_{+}^{+}++$

Sunitinib $(1 \mu \mathrm{M})++++++++$

Caspase $3-\infty=0-000$

cleaved- 3 a : 1 :

Caspase 3

pAKT-S473 - - $--1-60$

AKT $=0000-60$

OTUD1 $-\quad-\quad-{ }^{60}$

PTEN $0-1-1-2$

GAPDH $-\infty-37$ 786-O ACHN

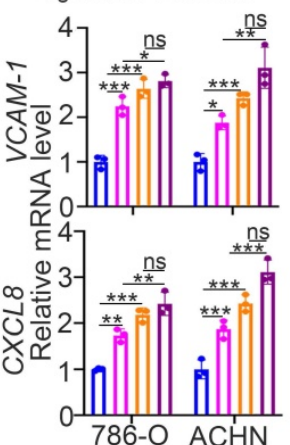

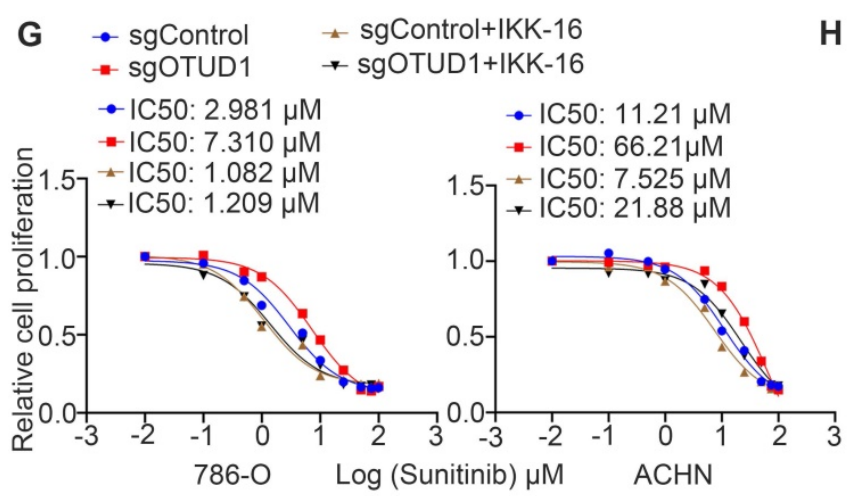

H

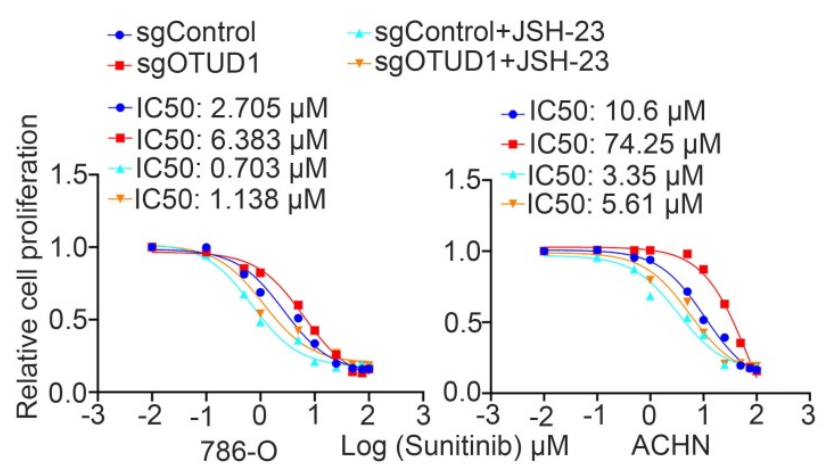

I - sgControl $\neq$ sgControl+MK2206

-sgOTUD1 -sgOTUD1+MK2206

- IC50: $2.319 \mu \mathrm{M}$

은 - IC50: $6.432 \mu \mathrm{M}$
윤 1.57 - IC50: $0.143 \mu \mathrm{M}$
- IC50: $0.228 \mu \mathrm{M}$

- IC50: $10.6 \mu \mathrm{M}$

- IC50: $74.25 \mu \mathrm{M}$

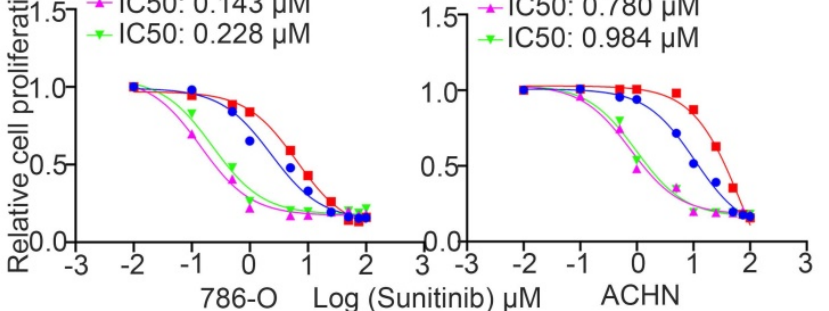

$J$
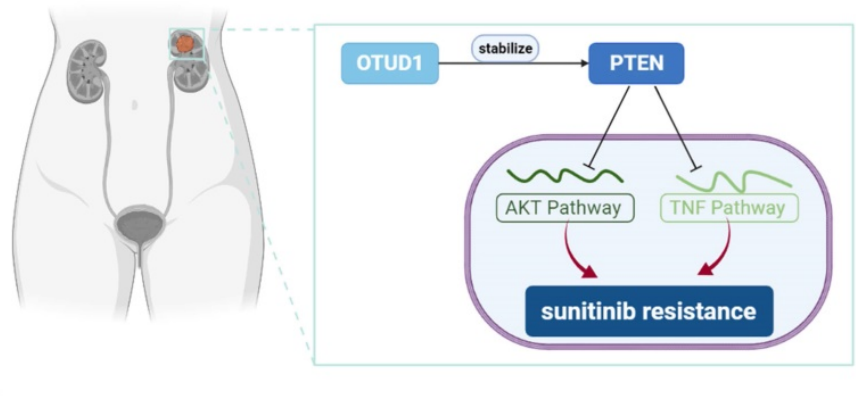

Figure 6. OTUDI sensitizes ccRCC cells to the TKIs via PTEN. A, 786-O and ACHN cells were transfected with indicated constructs for 48 h. Cells were treated a serial concentration of Sunitinib for measuring the IC50 values of Sunitinib. B, 786-O and ACHN cells were infected with indicated constructs for $72 \mathrm{~h}$. Cells were treated a serial 
concentration of Sunitinib for measuring the IC50 values of Sunitinib. C and D, 786-O and ACHN cells were transfected with indicated constructs for $48 \mathrm{~h}$. Cells were treated with $1 \mu \mathrm{M}$ Sunitinib for another $24 \mathrm{~h}$ and cells were harvested for Western blot (C) and RT-qPCR analysis (D). Data presents as mean \pm SD with three replicates. Ns, not significant; *, P $<0,05$; **, P $<0.01$; ***, P $<0.001$. E and F, 786-O and ACHN cells were infected with indicated constructs for $72 \mathrm{~h}$. Cells were treated with $1 \mu \mathrm{M}$ Sunitinib for another $24 \mathrm{~h}$ and cells were harvested for Western blot $(\mathrm{E})$ and RT-qPCR analysis $(\mathrm{F})$. Data presents as mean \pm SD with three replicates. Ns, not significant; *, P < 0,$05 ; * *, P<$ 0.01 ; ***, P $<0.001$. G, 786-O and ACHN cells were infected with indicated constructs for $72 \mathrm{~h}$. Then, these cells were pretreated with or without IKK-16 (500 nM) and treated a serial concentration of Sunitinib for measuring the IC50 values of Sunitinib. $\mathbf{H}, 786-\mathrm{O}$ and ACHN cells were infected with indicated constructs for $72 \mathrm{~h}$. Then, these cells were pretreated with or without $\mathrm{SSH}-23(10 \mu \mathrm{M})$ and treated a serial concentration of Sunitinib for measuring the IC50 values of Sunitinib. I, 786-O and ACHN cells were infected with indicated constructs for $72 \mathrm{~h}$. Then, these cells were pretreated with or without MK2206 (10 $\mu \mathrm{M})$ and treated a serial concentration of Sunitinib for measuring the IC50 values of Sunitinib. J, a model depicting that the downregulation of OTUDI stabilizes PTEN to inactivate AKT and TNF/NF-kappa B pathway and be responsible for the Sunitinib resistance in $\mathrm{ccRCC}$.

OTUD1 plays a key role in modulating the activation of AKT and TNF/NF-kappa B signaling in cCRCC. PTEN acts as a negative regulator of the AKT and TNF/NF-kappa B signaling pathways [13], and abnormal activation of AKT and TNF/NF-kappa B signaling contributes to TKI resistance in ccRCC [30, 31]. Thus, we aimed to study whether the effect of OTUD1 on the sensitivity of renal cancer cells to TKIs is mediated by the AKT and TNF/NF-kappa B signaling pathways. 786-O and ACHN cells were transfected with sgControl or sgOTUD1 and pretreated with TNF/NF-kappa B pathway and AKT pathway inhibitors (Fig. 6G-I). Then, the IC50 values of sunitinib were assessed. We demonstrated that IKK-16, JSH-23, and MK2206 attenuated the increase in the IC50 of sunitinib induced by OTUD1 ablation in both 786-O and ACHN cells (Fig. 6G-I). Thus, our results suggest that OTUD1 is involved in modulating the sensitivity of ccRCC to TKIs via PTEN (Fig. 6J).

\section{Discussion}

Studies have mentioned that OTUD1 plays a key role in modulating the immune response to viral infection. For instance, OTUD1 increases Smurf1 expression levels to promote degradation of the MAVS/TRAF3/TRAF6 signalosome and inhibit the innate immune response to viral infection [32]. OTUD1 deubiquitinates CARD9 to modulate antifungal innate immunity [33]. Meanwhile, OTUD1 is lost in multiple types of human cancer [22] and closely associated with cell survival and apoptosis. It has been well documented that OTUD1 deubiquitinates AIF at K244 to disrupt the mitochondrial structure and deubiquitinates AIF at K255 to promote its DNA-binding activity, which activates the caspaseindependent apoptotic pathway [34]. On the other hand, OTUD1 increases the stability of DCAF10 and DDB1 to activate the caspase-independent apoptotic pathway in cells [34]. However, $\mathrm{Wu}$ et al. reported that OTUD1 stabilizes MCL1 to block BH3-mimetic inhibitor-induced cell death in some types of cancer, including ovarian cancer, liver cancer and cervical cancer [35]. Moreover, Zhang et al. reported that OTUD1 binds with and stabilizes SMAD7 to repress the metastasis of breast cancer [22]. Here, we showed that low expression of OTUD1 is involved in the activation of the PI3K/AKT and TNF-alpha/
NF-kappa B signaling pathways by decreasing the stability of PTEN in ccRCC cells. Interestingly, OTUD3, another OTUD family member, regulates the stability of PTEN and repress tumorigenesis [36]. These findings indicated that OTUD1 and OTUD3 might share similar binding sites with PTEN. The mechanism of OTUD1 in regulating cancer progression is still unclear and needs to be further studied.

TKIs have been proven to be a promising therapeutic strategy and are currently recognized as the first-line treatment for ccRCC [37]. With the prolongation of the survival time of patients with ccRCC, acquired resistance to TKIs is the major factor determining the OS time of these patients [38]. Studies have reported that sunitinib treatment activates many resistance-associated pathways in renal cancer cells, such as the pERK-induced endoplasmic reticulum stress response pathway, PI3K/AKT signaling pathway, and NF-kappa B pathway [29]. The pERK-induced endoplasmic reticulum stress response upregulates the expression of IL-6, IL-8, and TNF- $a$ [29]. Blockade of pERK or administration of IL-6 neutralizing antibody or NF-kappa B inhibitors could restore the sensitivity of renal cancer cells to sunitinib $[29,31]$. In this study, we reanalyzed a TKI resistance dataset (GSE76068) and found that TNF and the NF-kappa B signaling pathway are very closely associated with sunitinib resistance in renal cancer. Treatment with TNF and NF-kappa B inhibitors sensitized renal cancer cells to sunitinib. Thus, the NF-kappa B pathway is an attractive therapeutic target for overcoming sunitinib resistance in ccRCC [19]. The AKT signaling pathway is also important for modulating the sensitivity of renal cancer cells to TKIs, and combination with AKT inhibitor treatment improved the anticancer effect of TKIs in ccRCC [28, 39]. Here, we showed that OTUD1 loss triggers the AKT and NF-kappa B signaling pathways and promotes TKI resistance partially by stabilizing PTEN in ccRCC. However, we believe that there must be other molecular mechanisms regulating TKI resistance mediated by OTUD1. Therefore, further studies are needed.

Collectively, we demonstrated that OTUD1 is downregulated in renal cancer and involved in the poor prognosis of renal cancer. Then, we showed that 
OTUD1 inhibits cancer cell growth. Moreover, analysis of OTUD1 RNA-seq data indicated that OTUD1 inhibition triggers the AKT and NF-kappa B pathways in renal cancer cells. Furthermore, OTUD1 interacts with PTEN and regulates its stability. Subsequently, we revealed that downregulation of OTUD1 contributes to the sensitivity of renal cancer cells to TKIs, and this effect was blocked by TNF/NF-kappa B inhibitors and AKT inhibitors. Thus, we identified that the OTUD1-PTEN axis suppresses tumor growth and regulates the resistance of renal cancer to TKIs.

\section{Abbreviations}

OTUD1: OTU domain-containing protein 1; PTEN: Phosphatidylinositol 3,4,5-trisphosphate 3-phosphatase and dual-specificity protein phosphatase; TKI: Tyrosine kinase inhibitor; RCC: Renal cell carcinoma; ccRCC: Clear cell renal cell carcinoma; TCGA: The Cancer Genome Atlas; KIRC: Kidney renal clear cell carcinoma.

\section{Supplementary Material}

Supplementary methods, figure and tables. https://www.ijbs.com/v18p1401s1.pdf

\section{Acknowledgements}

\section{Funding}

This work was supported by grants from the Hunan Provincial Development and Reform Commission (Grant No.2019875, 2021319 (L.Y.)); Hunan Provincial Health Commission (Grant No. 20200816 (L.Y.)); Department of Science and Technology of Hunan Province (Grant No. 2020SK2082 (L.Y.)).

\section{Author Contributions}

Xin Jin: Methodology, Writing - Original Draft, Project administration; Lu Yi: Methodology, Writing Original Draft, Project administration; Yinghuai Wang: Project administration; Liang Zhu: Conceptualization; Mou Peng: Methodology; Jiannan Ren: Methodology; Haixin Yu: Methodology; Bin Yan: Methodology, Investigation; Wentao Liu: Methodology, Investigation.

\section{Ethical Approval and Consent to participate}

The study was conducted in accordance with the principles of the Declaration of Helsinki principles. It was approved by the Animal Use and Care Committees at Second Xiangya hospital, Central south university.

\section{Consent for publication}

All subjects have written informed consent.

\section{Availability of supporting data}

The datasets used and/or analyzed during the current study are available from the corresponding authors (yilu9999@csu.edu.cn) on reasonable request.

\section{Competing Interests}

The authors have declared that no competing interest exists.

\section{References}

1. Hemminki O, Perlis N, Bjorklund J, Finelli A, Zlotta AR, Hemminki A. Treatment of Advanced Renal Cell Carcinoma: Immunotherapies Have Demonstrated Overall Survival Benefits While Targeted Therapies Have Not. Eur Urol Open Sci. 2020; 22: 61-73.

2. Siegel RL, Miller KD, Fuchs HE, Jemal A. Cancer Statistics, 2021. CA Cancer J Clin. 2021; 71: 7-33.

3. Shi L, Wang M, Li H, You P. MicroRNAs in Body Fluids: A More Promising Biomarker for Clear Cell Renal Cell Carcinoma. Cancer Manag Res. 2021; 13: 7663-75.

4. Brown LC, Zhang T, George DJ. The Role of Targeted Therapy in the Management of High-Risk Resected Kidney Cancer: What Have We Learned and How Will It Inform Future Adjuvant Trials. Cancer J. 2020; 26: 376-81.

5. Makhov P, Joshi S, Ghatalia P, Kutikov A, Uzzo RG, Kolenko VM. Resistance to Systemic Therapies in Clear Cell Renal Cell Carcinoma: Mechanisms and Management Strategies. Mol Cancer Ther. 2018; 17: 1355-64.

6. Tung I, Sahu A. Immune Checkpoint Inhibitor in First-Line Treatment of Metastatic Renal Cell Carcinoma: A Review of Current Evidence and Future Directions. Front Oncol. 2021; 11: 707214.

7. Jonasch E, Gao J, Rathmell WK. Renal cell carcinoma. BMJ. 2014; 349: g4797.

8. Yang DC, Chen CH. Potential New Therapeutic Approaches for Renal Cell Carcinoma. Semin Nephrol. 2020; 40: 86-97.

9. Molina AM, Lin X, Korytowsky B, Matczak E, Lechuga MJ, Wiltshire R, et al. Sunitinib objective response in metastatic renal cell carcinoma: analysis of 1059 patients treated on clinical trials. Eur J Cancer. 2014; 50: 351-8.

10. Shen J, Fu B, Li Y, Wu Y, Sang H, Zhang H, et al. E3 Ubiquitin Ligase-Mediated Regulation of Osteoblast Differentiation and Bone Formation. Front Cell Dev Biol. 2021; 9: 706395.

11. Tanguturi $P$, Kim KS, Ramakrishna S. The role of deubiquitinating enzymes in cancer drug resistance. Cancer Chemother Pharmacol. 2020; 85: 627-39.

12. Deng J, Hou G, Fang Z, Liu J, Lv XD. Distinct expression and prognostic value of OTU domain-containing proteins in non-small-cell lung cancer. Oncol Lett. 2019; 18: 5417-27.

13. Wu W, Jing D, Meng Z, Hu B, Zhong B, Deng X, et al. FGD1 promotes tumor progression and regulates tumor immune response in osteosarcoma via inhibiting PTEN activity. Theranostics. 2020; 10: 2859-71.

14. Yi L, Wang H, Li W, Ye K, Xiong W, Yu H, et al. The FOXM1/RNF26/p57 axis regulates the cell cycle to promote the aggressiveness of bladder cancer. Cell Death Dis. 2021; 12: 944.

15. Jin X, Ding D, Yan Y, Li H, Wang B, Ma L, et al. Phosphorylated RB Promotes Cancer Immunity by Inhibiting NF-kappaB Activation and PD-L1 Expression. Mol Cell. 2019; 73: 22-35 e6.

16. Li M, Wu W, Deng $\mathrm{S}$, Shao Z, Jin X. TRAIP modulates the IGFBP3/AKT pathway to enhance the invasion and proliferation of osteosarcoma by promoting KANK1 degradation. Cell Death Dis. 2021; 12: 767.

17. Piao S, Pei HZ, Huang B, Baek SH. Ovarian tumor domain-containing protein 1 deubiquitinates and stabilizes p53. Cell Signal. 2017; 33: 22-9.

18. Yao F, Zhou Z, Kim J, Hang Q, Xiao Z, Ton BN, et al. SKP2- and OTUD1-regulated non-proteolytic ubiquitination of YAP promotes YAP nuclear localization and activity. Nat Commun. 2018; 9: 2269.

19. Chen SL, Huang QS, Huang YH, Yang X, Yang MM, He YF, et al. GYS1 induces glycogen accumulation and promotes tumor progression via the NF-kappaB pathway in Clear Cell Renal Carcinoma. Theranostics. 2020; 10: 9186-99.

20. Ha SE, Kim SM, Vetrivel P, Kim HH, Bhosale PB, Heo JD, et al. Inhibition of Cell Proliferation and Metastasis by Scutellarein Regulating PI3K/Akt/NF-kappaB Signaling through PTEN Activation in Hepatocellular Carcinoma. Int J Mol Sci. 2021; 22.

21. Mevissen TE, Hospenthal MK, Geurink PP, Elliott PR, Akutsu M, Arnaudo N, et al. OTU deubiquitinases reveal mechanisms of linkage specificity and enable ubiquitin chain restriction analysis. Cell. 2013; 154: 169-84.

22. Zhang Z, Fan Y, Xie F, Zhou H, Jin K, Shao L, et al. Breast cancer metastasis suppressor OTUD1 deubiquitinates SMAD7. Nat Commun. 2017; 8: 2116.

23. Han P, Li H, Jiang X, Zhai B, Tan G, Zhao D, et al. Dual inhibition of Akt and c-Met as a second-line therapy following acquired resistance to sorafenib in hepatocellular carcinoma cells. Mol Oncol. 2017; 11: 320-34.

24. Piovan E, Yu J, Tosello V, Herranz D, Ambesi-Impiombato A, Da Silva AC, et al. Direct reversal of glucocorticoid resistance by AKT inhibition in acute lymphoblastic leukemia. Cancer Cell. 2013; 24: 766-76. 
25. Wang B, Zhang W, Zhang G, Kwong L, Lu H, Tan J, et al. Targeting mTOR signaling overcomes acquired resistance to combined BRAF and MEK inhibition in BRAF-mutant melanoma. Oncogene. 2021; 40: 5590-9.

26. George DJ, Lee $\mathrm{CH}$, Heng D. New approaches to first-line treatment of advanced renal cell carcinoma. Ther Adv Med Oncol. 2021; 13: 17588359211034708 .

27. Armesto M, Marquez M, Arestin M, Errarte P, Rubio A, Manterola L, et al Integrated mRNA and miRNA Transcriptomic Analyses Reveals Divergent Mechanisms of Sunitinib Resistance in Clear Cell Renal Cell Carcinoma (ccRCC). Cancers (Basel). 2021; 13

28. Xiong $\mathrm{W}$, Zhang B, Yu H, Zhu L, Yi L, Jin X. RRM2 Regulates Sensitivity to Sunitinib and PD-1 Blockade in Renal Cancer by Stabilizing ANXA1 and Activating the AKT Pathway. Adv Sci (Weinh). 2021; 8: e2100881.

29. Makhov P, Naito S, Haifler M, Kutikov A, Boumber Y, Uzzo RG, et al. The convergent roles of NF-kappaB and ER stress in sunitinib-mediated expression of pro-tumorigenic cytokines and refractory phenotype in renal cell carcinoma. Cell Death Dis. 2018; 9: 374.

30. Peng D, Hu Z, Wei X, Ke X, Shen Y, Zeng X. NT5E inhibition suppresses the growth of sunitinib-resistant cells and EMT course and AKT/GSK-3beta signaling pathway in renal cell cancer. IUBMB Life. 2019; 71: 113-24.

31. Zhu $Y$, Liu $H, X_{\text {u L }}$, An $H_{\text {, Liu }} W$, Liu $Y$, et al p21-activated kinase 1 determines stem-like phenotype and sunitinib resistance via NF-kappaB/IL-6 activation in renal cell carcinoma. Cell Death Dis. 2015; 6: e1637.

32. Zhang L, Liu J, Oian L, Feng $\mathrm{O}$, Wang X, Yuan $Y$, et al. Induction of OTUD1 by RNA viruses potently inhibits innate immune responses by promoting degradation of the MAVS/TRAF3/TRAF6 signalosome. PLoS Pathog. 2018; 14: e1007067.

33. Chen X, Zhang H, Wang X, Shao Z, Li Y, Zhao G, et al. OTUD1 Regulates Antifungal Innate Immunity through Deubiquitination of CARD9. J Immunol. 2021; 206: 1832-43.

34. Luo $\mathrm{O}, \mathrm{Wu} X$, Zhao $P$, Nan $Y$, Chang $W$, Zhu $X$, et al, OTUD1 Activates Caspase-Independent and Caspase-Dependent Apoptosis by Promoting AIF Nuclear Translocation and MCL1 Degradation. Adv Sci (Weinh). 2021; 8: 2002874.

35. Wu L, Lin $Y$, Feng J, Qi Y, Wang X, Lin Q, et al. The deubiquitinating enzyme OTUD1 antagonizes BH3-mimetic inhibitor induced cell death through regulating the stability of the MCL1 protein. Cancer Cell Int. 2019; 19: 222.

36. Yuan L, Lv Y, Li H, Gao H, Song S, Zhang Y, et al. Deubiquitylase OTUD3 regulates PTEN stability and suppresses tumorigenesis. Nat Cell Biol. 2015; 17: $1169-81$

37. Tegos T, Tegos K, Dimitriadou A, Dimitriadis G. Current and emerging first-line systemic therapies in metastatic clear-cell renal cell carcinoma. J BUON. 2019; 24: 1340-53.

38. Kamli H, Li L, Gobe GC. Limitations to the Therapeutic Potential of Tyrosine Kinase Inhibitors and Alternative Therapies for Kidney Cancer. Ochsner J. 2019; 19: 138-51.

39. Miricescu D, Balan DG, Tulin A, Stiru O, Vacaroiu IA, Mihai DA, et al. $\mathrm{PI} 3 \mathrm{~K} / \mathrm{AKT} / \mathrm{mTOR}$ signalling pathway involvement in renal cell carcinoma pathogenesis (Review). Exp Ther Med. 2021; 21: 540. 